\title{
State-specific transport properties of partially ionized flows of electronically excited atomic gases
}

\author{
V.A. Istomin*, E.V. Kustova \\ Saint Petersburg State University, 7/9 Universitetskaya nab., St. Petersburg 199034, Russia
}

\section{A R T I C L E I N F O}

\section{Article history:}

Received 28 December 2016

In final form 29 January 2017

Available online 2 February 2017

\begin{abstract}
A B S T R A C T
State-to-state approach for theoretical study of transport properties in atomic gases with excited electronic degrees of freedom of both neutral and ionized species is developed. The dependence of atomic radius on the electronic configuration of excited atoms is taken into account in the transport algorithm. Different cutoff criteria for increasing atomic radius are discussed and the limits of applicability for these criteria are evaluated. The validity of a Slater-like model for the calculation of state-resolved transport coefficients in neutral and ionized atomic gases is shown. For ionized flows, a method of evaluation for effective cross-sections of resonant charge-transfer collisions is suggested. Accurate kinetic theory algorithms for modelling the state-specific transport properties are applied for the prediction of transport coefficients in shock heated flows. Based on the numerical observations, different distributions over electronic states behind the shock front are considered. For the Boltzmann-like distributions at temperatures greater than $14,000 \mathrm{~K}$, an important effect of electronic excitation on the partial thermal conductivity and viscosity coefficients is found for both neutral and ionized atomic gases: increasing radius of excited atoms causes a strong decrease in these transport coefficients. Similarly, the presence of electronically excited states with increased atomic radii leads to reduced diffusion coefficients. Nevertheless the overall impact of increasing effective cross-sections on the transport properties just behind the shock front under hypersonic reentry conditions is found to be minor since the populations of high-lying electronic energy levels behind the shock waves are low.
\end{abstract}

(ㄷ) 2017 Elsevier B.V. All rights reserved.

\section{Introduction}

For the long time the influence of electronic degrees of freedom on transport properties was out of consideration with a few exceptions for the pioneering works by Hirschfelder [1] and Capitelli [2]. The reason for such a neglect is complexity of theoretical modelling and high computational efforts for its implementation. This gap in the transport kinetic theory starts to filling out during the last quarter-century. Algorithms for the calculation of transport coefficients in chemically equilibrium plasmas and one-temperature non-equilibrium partially ionized gases were proposed [311]. It was shown that in high-temperature flows, neglecting electronically excited species yields considerably under-predicted convective heat flux.

The objective of the present study is to generalize our previous one- temperature models of high-temperature reacting flows with electronic excitation $[10,12-15]$ by including state-to-state kinetics of electronic levels and state-dependent transport coefficients. While the state-to-state model has been widely used in simula-

\footnotetext{
* Corresponding author.
}

tions of vibrationally excited flows (see references in $[16,11]$ ), its implementation for gases with electronic excitation is just starting $[17,18,11,19,20]$. In Ref. [11], a near-equilibrium plasma of molecular and atomic hydrogen with electronically excited states of neutral atoms was studied in the state-to-state approach, and the contribution of electronic levels to the thermal conductivity coefficient was found to be important. Nevertheless, systematic studies of state-resolved transport properties in electronically excited gases are still missing. Moreover, evaluation of the influence of collision diameters of many-electron atoms on the transport properties in atomic electronically excited gases is still an open problem.

In the present paper a detailed state-to-state model for a nonequilibrium high-temperature atomic plasma flow of neutral $N$ and ionized $N / N^{+} / e^{-}$atomic nitrogen, and neutral $O$ and ionized $O / O^{+} / e^{-}$oxygen, taking into account respectively 170 and 204 electronic energy levels for neutral $N$ and $O$, and 157 and 254 for ionized $\mathrm{N}^{+}$and $\mathrm{O}^{+}$, is developed on the basis of the modified Chapman-Enskog method. The approach proposed in the present study makes it possible to evaluate transport terms directly, with no separation of low-lying and high-lying electronically excited states $[21,22,11]$. The distribution of atomic species over electronic 
energy levels may play an important role for strongly non-equilibrium flows, therefore we calculate the transport properties on the basis of different state-to-state distributions obtained in $[21,22]$. For the evaluation of transport properties, conditions characteristic for the spacecraft re-entry (Fire II experiments [23]) into the planetary atmosphere are chosen. The influence of increasing atomic radius on transport coefficients of gases with electronically excited states is discussed.

The paper has the following structure: in the next section, macroscopic fluid dynamic variables and governing equations for them are introduced according to the state-to-state flow description. These equations are suitable for non-equilibrium high-temperature reacting viscous flows of ionized atomic gases with arbitrary distributions of species over electronic states. Then the expressions for the transport terms as well as the algorithms for the calculation of state-resolved transport coefficients are derived. Further, in the Section 4, the methods of calculation of the atomic radius and effective collision cross-section of electronically excited atoms are discussed. Special focus is on the possibility of implementation of the Slater's approach for the evaluation of atomic radius and effective cross-sections of the collisions of neutral-neutral and neutral-charged species (the case of resonant charge-transfer process). The limits of applicability of various cutoff criteria are indicated, and the maximum allowed atomic radius is calculated for different initial conditions. Finally, in the Sections 5 and 6, different state-to-state distributions and corresponding state-resolved transport coefficients (partial thermal conductivity, shear viscosity, diffusion and thermal diffusion) are calculated, and the effects of the number of accounted electronic levels and the state-dependent atomic radius on the transport terms are estimated.

\section{Macroscopic parameters and governing equations}

We consider non-equilibrium high-temperature reacting flows of ionized atomic gases under the following relation between characteristic times of collisional processes:

$\tau_{\text {ch-tr }} \sim \tau_{\text {tr }} \ll \tau_{E T} \sim \tau_{\text {ioniz }} \sim \theta$,

here $\tau_{c h-t r}, \tau_{t r}, \tau_{E T}$ and $\tau_{\text {ioniz }}$ are the characteristic times for resonant charge-exchange due to particle collisions, for translational relaxation, for electronic energy relaxation and ionization, respectively; $\theta$ is the mean time of the variation of gas-dynamic parameters. Under such an assumption, relaxation of electronic energy proceeds at the macroscopic time scale and the state-to-state modeling of kinetics and transport properties is to be implemented [16].

The closed set of governing equations for the macroscopic parameters taking into account electronic degrees of freedom of atoms is derived from the kinetic equations for distribution function using the generalized Chapman-Enskog method [16]. The kinetic equations for the distribution function are multiplied by the collision invariants, which are represented by the momentum, particle total energy as well as the additional invariants of the most frequent collisions (which are independent of the velocity and depend arbitrarily on the electronic level and chemical species), integrated over velocity and summed over electronic levels and chemical species. In the case of the absence of external forces and magnetic fields the governing equations are obtained in the following form:

$\frac{d \mathrm{n}_{c_{n}}}{d t}+\mathrm{n}_{c_{n}} \nabla \cdot \mathbf{v}+\nabla \cdot\left(\mathrm{n}_{c_{n}} \mathbf{V}_{c_{n}}\right)=R_{c_{n}}^{E T}+R_{c_{n}}^{\text {ioniz }}$

$c=X, X^{+}, e^{-}, n=1, \ldots, L_{c}$,

$\rho \frac{d \mathbf{v}}{d t}+\nabla \cdot \boldsymbol{P}=0$ $\rho \frac{d U}{d t}+\nabla \cdot \mathbf{q}+\boldsymbol{P}: \nabla \mathbf{v}=0$

here $\mathrm{n}_{c_{n}}$ is the number density of atom $c$ on the electronic level $n$ with energy $\varepsilon_{c_{n}}, \mathbf{v}$ is the gas velocity, $L_{c}$ is the number of accounted levels for $c$-species, $X$ is the corresponding atom $N$ or $O, U$ is the total specific energy including the electronic one, $\rho U=\frac{3}{2} n k T+\sum_{c, n} \varepsilon_{n} n_{c_{n}}+\sum_{c, n} E_{i_{c}} n_{c_{n}} ; E_{i_{c}}$ is ionization energy, $\mathbf{V}_{c_{n}}$ is the diffusion velocity of $c$-species for each electronic state $n, R_{c_{n}}^{E T}$ and $R_{c_{n}}^{\text {ioniz }}$ are the production terms due to electronic energy transitions and ionization, $\rho$ is the mixture density, $\boldsymbol{P}$ is the pressure tensor, $\mathbf{q}$ is the heat flux. Eqs. (2) include conservation equations coupled with equations of detailed electronic state and chemical (ionization) kinetics. While a similar state-to-state approach is widely used for modeling vibrational relaxation (see references in $[16,11])$, it is quite novel for the description of electronically excited gases. It is worth noting that it is not easy to implement this model into the computational fluid dynamics due to considerable computational efforts and absence of data of transition probabilities between electronic energy levels. On the other hand such a detailed description of the electronic state kinetics is rather promising for correct predictions of the heat and mass transfer in high-temperature flows.

The expressions for the pressure tensor, diffusion velocity, and heat flux in a viscous flow taking into account state-resolved transport coefficients can be derived using the procedure of the modified Chapman-Enskog method:

$\boldsymbol{P}=p \boldsymbol{I}-2 \eta \boldsymbol{S}$,

$\mathbf{V}_{c_{n}}=-\sum_{d, m} D_{c_{n} d_{m}} \mathbf{d}_{c_{n}}-D_{T c_{n}} \nabla \ln T$

$\mathbf{q}=-\lambda^{\prime} \nabla T-p \sum_{c, n} D_{T c_{n}} \mathbf{d}_{c_{n}}+\sum_{c, n}\left(\frac{5}{2} k T+\varepsilon_{c_{n}}+E_{i_{c}}\right) \mathrm{n}_{c_{n}} \mathbf{v}_{c_{n}}$.

Here $\boldsymbol{S}$ and $\boldsymbol{I}$ are deformation rate and unit tensors, $\mathbf{d}_{c_{n}}$ is the diffusive driving force for each electronic state of $c$ species:

$\mathbf{d}_{c_{n}}=\nabla\left(\frac{\mathrm{n}_{c_{n}}}{\mathrm{n}}\right)+\left(\frac{\mathrm{n}_{c_{n}}}{\mathrm{n}}-\frac{\rho_{c_{n}}}{\rho}\right) \nabla \ln p$,

$p, T$ are the pressure and temperature, $\eta$ is the shear viscosity coefficient, $D_{c_{n} d_{m}}, D_{T c_{n}}$ are the diffusion and thermal diffusion coefficients for different electronic states, $\lambda^{\prime}$ is the partial thermal conductivity coefficient. In the present case, normal mean stress does not include bulk viscosity and relaxation pressure, which are specified by rapid inelastic processes and appear in the one-temperature model [16]. This can be explained by the fact that under relation (1) all rapid processes are either elastic or resonant. Moreover, in the state-to-state approach the thermal conductivity coefficient is specified only by translational degrees of freedom, whereas the transport of electronic energy is governed by diffusion processes and therefore can be strongly affected by non-equilibrium kinetics of electronic states. The last but not least in the comparison of these two approaches is that in the one-temperature model for pure atomic gases ( $c=d$ in the model below) thermal and mass diffusion vanish $\left(D_{c_{n} d_{m}}=D_{T c_{n}}=0\right)$, which is not the case for Eqs. (6) and (7) where $D_{c_{n} c_{m}}$ and $D_{T c_{n}} \neq 0$ for a single-component gas. It is interesting to emphasize that in some sense, the state-tostate approach for atomic gases with electronic excitation is similar to the model of vibrationally excited molecular gas under condition of slow vibrational relaxation. In the present model, kinetics of electronic states is investigated instead of that for vibrationally excited levels. 


\section{Transport coefficients}

Using the Chapman-Enskog procedure the transport coefficients are derived by reducing integral equations for the first-order distribution function to the systems of linear algebraic equations using expansions of unknown functions into the series of Sonine polynomials. It is worth noting, that due to the slow convergence of Sonine polynomials for the case of ionized gases it is not sufficient to keep in the expansions only the first non-vanishing terms. In the present study, the first three terms: $r=0 . .2$, are retained. In terms of the expansion coefficients $b_{c_{n}, r}, a_{c_{n}, r}, d_{c_{n}, r}^{d_{m}}$ the expressions for the transport coefficients take the following form:

$\eta=\frac{k T}{2} \sum_{c_{n}} \frac{\mathrm{n}_{c_{n}}}{\mathrm{n}} b_{c_{n}, 0}, \quad \lambda^{\prime}=\sum_{c, n} \frac{5}{4} k \frac{\mathrm{n}_{c_{n}}}{\mathrm{n}} a_{c_{n}, 1}$,

$D_{T c_{n}}=-\frac{1}{2 \mathrm{n}} a_{c_{n}, 0}, \quad D_{c_{n} d_{m}}=\frac{1}{2 \mathrm{n}} d_{c_{n}, 0}^{d_{m}}$

The transport linear systems for the expansion coefficients are derived similarly to [16]. Thus, for the shear viscosity coefficient it is necessary to find the first coefficient $b_{(c, n), 0}$ of the system

$\sum_{d, m} H_{00}^{c_{n} d_{m}} b_{d_{m}, 0}=\frac{2}{k T} \frac{\mathrm{n}_{c_{n}}}{\mathrm{n}}, \quad c=X, X^{+}, e^{-}, n=1, \ldots, L_{c}$,

$H_{00}^{c_{n} d_{m}}$ is the bracket integral specified by the cross-sections of rapid processes [16]; in the present case they include only elastic collisions and charge transfer.

Partial thermal conductivity and thermal diffusion are found from the system for the coefficients $a_{c_{n}, r}$ supplemented by the constraint required to obtain the unique solution:

$$
\begin{aligned}
& \sum_{d, m} \sum_{r^{\prime}} \Lambda_{r r^{\prime}}^{c_{n} d_{m}} a_{d_{m}, r^{\prime}}=\frac{15 k T}{2} \frac{\mathrm{n}_{c_{n}}}{\mathrm{n}} \delta_{r 1}, \\
& c=X, X^{+}, e^{-}, \quad n=1 \ldots L_{c}, \quad r=0,1, \ldots, \\
& \sum_{c, n} \frac{\rho_{c_{n}}}{\rho} a_{c_{n}, 0}=0,
\end{aligned}
$$

with $\Lambda_{r r^{\prime}}^{c_{n} d_{m}}$ being the corresponding bracket integrals.

Similarly, for diffusion coefficients we have:

$$
\begin{aligned}
& \sum_{d, m} \Lambda_{00}^{c_{n} d_{m}} d_{d_{m}, 0}^{b_{l}}=3 k T\left(\delta_{c_{n} b_{l}}-\frac{\rho_{c_{n}}}{\rho}\right), \\
& b, d=X, X^{+}, e^{-}, \quad l, m=1, \ldots, L_{b, d}, \\
& \sum_{c, n} \frac{\rho_{c_{n}}}{\rho} d_{c_{n}, 0}^{d_{m}}=0, \quad d=X, X^{+}, e^{-}, \quad l=1, \ldots, L_{d} .
\end{aligned}
$$

The bracket integrals, after some transformations, can be expressed in terms of collision integrals $\Omega_{c_{n} d_{m}}^{(k, r)}$ calculated for each pair of excited electronic states $n$ and $m$ of atoms $c$ and $d$. In the present study we calculate the collision integrals using the models proposed in [24] but taking into account increasing atomic radius (i.e. effective cross-section) of electronically excited atoms. The transport coefficients are then calculated numerically as the solutions of the transport linear systems, and expressed in terms of the collision integrals.

\section{Radius and effective collision cross-section of electronically excited atom}

\subsection{Slater diameter and effective collision cross-section}

Collision integrals for electronically excited states depend significantly on the effective cross-section of colliding species [1]. On the other hand the effective cross-section is specified by the atomic radius, that is defined as a radius of the outermost electron on atomic orbital and depends on the principle quantum number [1]. For a hydrogen-like atom, this dependence for the outermost electron can be expressed directly through the square principle quantum number $n$ :

$r_{n}=\frac{4 \pi \hbar \varepsilon_{0}}{m_{e} c^{2}} n^{2} \approx a_{0} n^{2}$

here $\hbar$ is the Plank constant, $\varepsilon_{0}$ is the vacuum permittivity constant, $m_{e}$ is the mass of electron, $c$ is the speed of light, $a_{0}$ is the Bohr radius.

For atomic orbitals of many-electron atoms (in particular, $N, \mathrm{~N}^{+}, \mathrm{O}$, and $\mathrm{O}^{+}$, considered in the present study), no closed analytical form can be given because the orbital approximation is very primitive [25]. In the case of many-electron atoms, the actual wave-function can be found using a sophisticated numerical technique which helps to define the so-called Slater-type orbital belonging to a nucleus of an atom of the atomic number $Z$ [25]:

$\psi(r, \theta, \phi)=N r^{n^{*}-1} \exp ^{\varsigma r / a_{0}} Y_{l m_{l}}(\theta, \phi), \quad \varsigma=\frac{Z-S}{n^{*}}$,

where $N$ is the normalization constant, the effective principle quantum number $n^{*}$ is a function of the principal quantum number $n$ (see Table 1 ), $\varsigma$ is an orbital exponent of a single exponential function describing an atomic orbital, $S$ is the screening constant in the Slater orbital [26,27], $Y_{l m_{l}}$ is a spherical harmonic depending on the orbital quantum number $l$ and magnetic quantum number $m_{l}$. For ground electronic states, the values $Z-S$ have been constructed by fitting Slater-type orbitals to numerically computed wave-functions [25]. The latter values now replace those originally proposed by Slater in terms of several simple rules [27].

Slater's approach makes it possible to represent the collision diameter of colliding neutral atomic species through the semiempirical relation [1]:

$\sigma_{\text {Slater }}=2 r_{0}+1.8 \AA, \quad r_{0}=\frac{2 n^{*}+1}{2 \varsigma} a_{0}$,

where $r_{0}$ is the mean electron radius (i.e. atomic radius), and the screening constant $S$ for high-lying states should be calculated directly with the set of Slater's rules [27] mentioned above.

Based on the values for effective quantum number $n^{*}$, which is specified as a function of $n=1 \ldots 6$, for greater values of principal quantum number $n>6$ we constructed an analytical formula for high-lying electronic states:

$n_{h-l}^{*}=1.8886 \log n+0.9124$,

\begin{tabular}{|c|c|c|c|c|c|c|c|c|c|c|c|c|}
\hline$n$ & 1 & 2 & 3 & 4 & 5 & 6 & 7 & 8 & 9 & 10 & 11 & 12 \\
\hline$n^{*}$ & 1 & 2 & 3 & 3.7 & 4 & 4.2 & - & - & - & - & - & - \\
\hline$n_{h-l}^{*}$ & 0.91 & 2.22 & 2.99 & 3.53 & 3.95 & 4.30 & 4.59 & 4.84 & 5.06 & 5.26 & 5.44 & 5.61 \\
\hline
\end{tabular}

that yields the coefficient of determination close to one: $R^{2}=0.9877$.

Table 1

Quantum number $n$, effective quantum number $n^{*}$, and $n_{h-l}^{*}$ calculated using the analytical formula (19) for low and high-lying states. 
In the general case, various orbitals with different values of $n$ but the same values of $l$ and $m_{l}$ are not orthogonal; $n s$-orbitals with $n>1$ have a zero amplitude at the nucleus, and the Slater's formula should be used with care [25]. In the present study we do not account for these exceptions to the basic approximation.

It is known that collision integrals may considerably increase with the rise of principle quantum number $[1,11,13]$. It is however worth mentioning that implementation of the Slater's approach (18) yields over-predicted transport cross-section and does not account for the resonant processes of excitation and chargeexchange in collisions involving excited atoms or ion-parent atoms $[11,28]$. Moreover collision integrals that are handled by interaction potential also depend on polarization of each electronic state, and the cross-sections of these integrals should involve only allowed transitions for the electronic energy exchange. In the modern models $[29,24,11]$ of inter-particle potentials, a phenomenological approach is implemented. The phenomenological potentials $\varphi(x)$ are constructed combining the well-known Lennard-Jones and Born-Mayer-type potentials which are fitted to experimental results. For such potentials, the collision diameter is a product, $\sigma=x_{0} r_{e}$, where $x_{0}$ depends on the "softness" of colliding particles and corresponds to the minimum of the potential function $\varphi\left(x_{0}\right)=0 ; r_{e}$ is the parameter depending on the polarizability of colliding particles. Due to the absence of experimental data on polarizability and "softness" coefficients for each electronically excited state, we put $x_{0}, r_{e}$ equal to those in the ground electronic state. Whereas the last assumption is a rough approximation for different electronically excited states, we should mention that for the low-lying levels of $\mathrm{N}, \mathrm{O}, \mathrm{N}^{+}$or $\mathrm{O}^{+}$the differences in the values for $r_{e}$ does not exceed $1 \%$, while the discrepancies in values for polarizability and parameters of $x_{0}$ can achieve $30 \%$. Concluding this subsection we should say that at the present moment, the dependence of collision integrals on the quantum state of chemical species is largely unknown and its determination still represents a challenging problem [11].

\subsection{Cutoff criteria for electronically excited states}

For modelling flows of electronically excited species, the assumption on the time between collisions, which is much shorter than the natural lifetime of these states, is needed [30]. For excited states of hydrogen-like atoms, the natural lifetime between absorption and re-emission rises as a cube of the principle quantum number multiplied by the lifetime of the ground electronic state: $\tau_{n}=\tau_{1} n^{3}$ (see Ref. [31]), so far excited states, especially high-lying, can be treated as an object possessing the lifetime comparable with the mean time of variation of gas dynamics parameters, and must be included into the kinetic scheme for atomic gas flows. For metastable excited states corresponding to forbidden transitions (i.e. forbidden by electric dipole selection rules) the lifetime may be even higher than that stated above. Consequently, excited states of atoms may exist at the macroscopic timescale.

On the other hand, accounting for this manifold of states is a comprehensive and complex computational task and still represents a challenging problem. Therefore a description of electronic excitation should include a discussion on the necessary number of accounted levels. Usually, the following cutoff criteria are implemented [32]:

1. The most often used and well known, "the ground-state model", that completely disregards the presence of electronically excited particles as well as their influence on lowering of the ionization potential.

2. The so called "confined atom approximation" assumes that the radius of electronically excited species does not exceed the inter-particle distance (i.e. mean free path). The confined atom approximation is usually represented by the expression (16) for the atomic radius of hydrogen-like atoms. This expression cannot be implemented for many-electron atoms like $\mathrm{N}, \mathrm{O}, \mathrm{N}^{+}$or $\mathrm{O}^{+}$, and more complicated analysis of atomic radii has to be done (estimates of this approach are provided below for different test cases at the temperature range $1000-60,000 \mathrm{~K}$ ). It is worth noting that the confined atom approximation takes into account neither the dependence of ionization potentials on the presence of excited particles nor the presence of electrons and ions in the mixture.

3. The most accurate results are obtained by using the DebyeHuckel criteria (or so called "static screened Coulomb potential" model), that truncates the series for the internal partition functions at the term corresponding to electronically excited levels of atoms with energy above the corrected ionization potential predicted by the Debye-Huckel theory:

$$
r_{\max }=Z_{\text {eff }} \lambda_{D},
$$

where $Z_{\text {eff }}$ is the effective charge seen by the electronic excited state, $z_{c}$ is the charge of the $c$-species ( 0 for neutrals, -1 for electrons, 1 for single-ionized etc.), $\lambda_{D}$ is the Debye length:

$$
\begin{aligned}
& Z_{\text {eff }}=z_{c}+1, \\
& \lambda_{D}=\sqrt{\frac{\varepsilon_{0} k T}{\sum_{c} n_{c} e_{c}^{2}}}, \quad c=1 \ldots L .
\end{aligned}
$$

In Eq. (22), $n_{c}$ and $e_{c}$ are the number density and the charge of n-species in the mixture of $L$ species.

In Fig. 1, the maximum allowed atomic radius for arbitrary electronically excited species in the mixture is presented as a function of temperature $T$ and pressure $p$ for the confined atom approximation and the Debye-Huckel criterion (in the latter case it depends also on the molar fraction of ionized species) under specific flow conditions. We assume the Debye-Huckel criterion to be the most accurate one for ionized mixtures, and we consider conditions corresponding to shock heated flows for two cases of re-entering spacecrafts: Hermes [33,34], and Fire II [23] at the trajectory point $1634 \mathrm{~s}$. The initial conditions before the shock front for Fire II $\left(v_{0}=11360 \mathrm{~m} / \mathrm{s}, \quad T_{0}=195 \mathrm{~K}, \quad p_{0}=2 \mathrm{~Pa}\right)$ yield the post-shock steady-state pressure $p_{1}=4200 \mathrm{~Pa}$ and the fraction of ionized species up to $5 \%$. For the Hermes case $\left(v_{0}=7198 \mathrm{~m} / \mathrm{s}, T_{0}=205 \mathrm{~K}\right.$, $p_{0}=2$ Pa before the shock front) the post-shock steady-state pressure is $p_{1}=2000 \mathrm{~Pa}$ and the fraction of ionized species does not exceed $2 \%$. In order to study the pressure effect on the atomic radius we include also $r_{\max }$ calculated at the atmospheric pressure. It is seen that the confined atom approximation essentially underestimates the maximum value of the atomic radius, especially at low pressure. Indeed, the presence of charged species in the mixture changes the type of interaction potential from the short-range to the electronically screened long-range (i.e. taking into account the long-range Coulomb forces) [35] thus enlarging the maximum allowed atomic radius. It is worth to note that for both cutoff criteria, the discrepancy between the maximum allowed radius calculated for different pressures remains approximately the same.

\subsection{Effective collision cross-section for collisions of neutral atoms}

In addition to the Slater's approach let us consider an attempt of Clementi [36] to refine the Slater's formula, proposing the formulae for the collision diameter for each electronic level as a function of $\varsigma$ depending on $Z$. In the Table 2 collision diameters calculated with the use of different models are given: (1) $\sigma_{1}$ for the Slater formula 

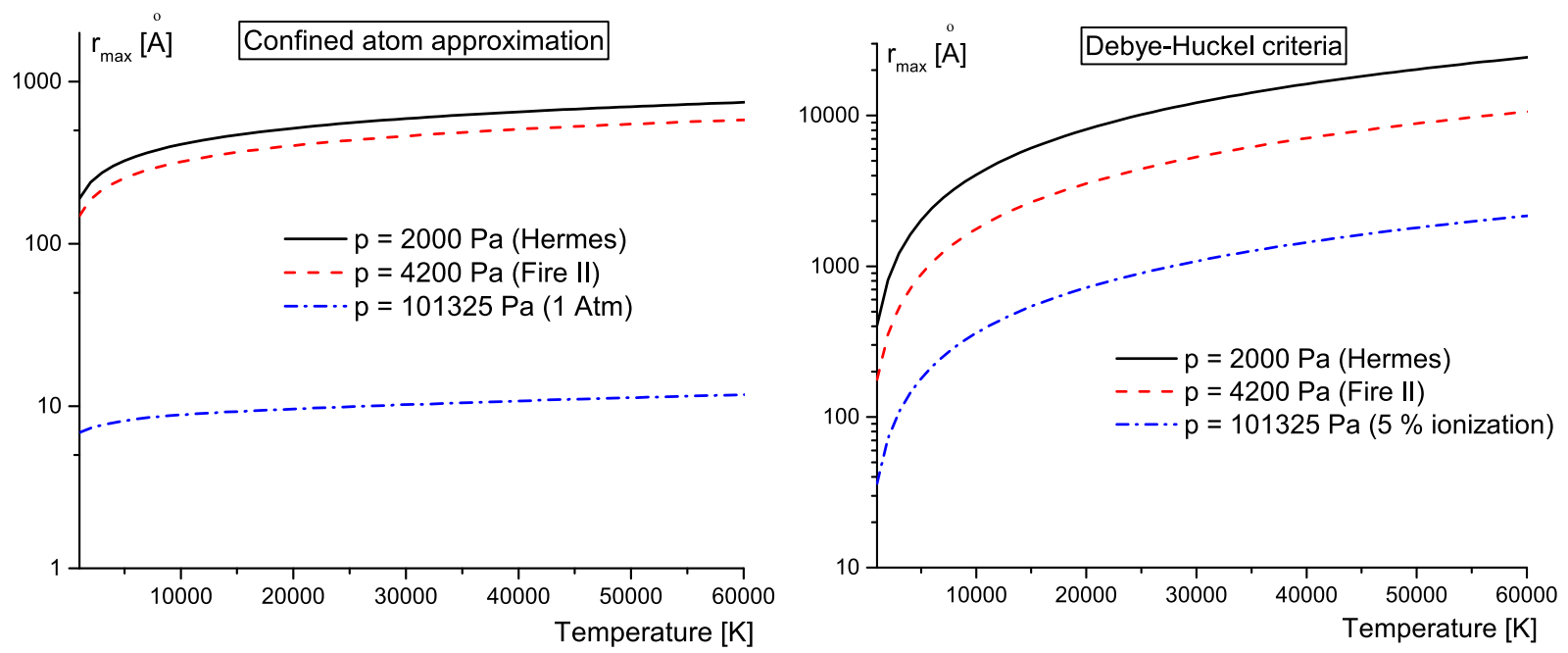

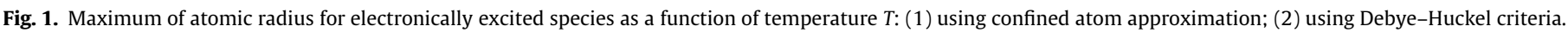

Table 2

Collision diameters of neutral and ionized atomic nitrogen and oxygen at the ground state calculated with different models.

\begin{tabular}{|c|c|c|c|c|c|}
\hline Interaction & $\sigma_{1},[\AA ̊]$ & $\left.\sigma_{2},[\AA]\right]$ & $\sigma_{3},[\AA]$ & $\sigma_{4},[\AA ̊]$ & $\frac{\sigma_{1}-\sigma_{4}}{\sigma_{4}} \cdot 100 \%[\%]$ \\
\hline$N(4 S)-N(4 S)$ & 3.15686 & 3.52129 & 3.18022 & 3.14663 & 0.32 \\
\hline$O(3 P)-O(3 P)$ & 2.96303 & 3.72723 & 2.98831 & 3.01248 & -1.64 \\
\hline$N(4 S)-N^{+}(3 P)$ & 2.60198 & $\mathrm{n} / \mathrm{a}$ & $\mathrm{n} / \mathrm{a}$ & 2.52973 & 2.86 \\
\hline$O(3 P)-O^{+}(4 S)$ & 2.24298 & $\mathrm{n} / \mathrm{a}$ & $\mathrm{n} / \mathrm{a}$ & 2.30493 & -2.69 \\
\hline
\end{tabular}

[27]; (2) $\sigma_{2}$ for the Clementi formula [36]; (3) $\sigma_{3}$ for the modified Slater formula for the ground state collision diameter with the Clementi's data for the orbital exponent $\varsigma$; (4) $\sigma_{4}$ proposed in [24], and given in the form $\sigma=x_{0} r_{e}$ (see discussion in Section 4.1). The latter data are considered as the most reliable. In [24], collision integrals obtained using the same data are compared with the accurate results reported in $[37,38]$, and a good agreement is shown (the relative error is within $6 \%$ ).

It is seen that $\sigma_{2}$ exceeds the other ones up to $12 \%$ for $N$ and $23.7 \%$ for $O$ (compared to the most reliable data $\sigma_{4}$ ). On the other hand, the discrepancy between $\sigma_{1}$ and $\sigma_{3}$ with respect to $\sigma_{4}$ is small: corresponding errors do not exceed $0.3 \%$ and $1 \%$ for $N$, and $1.7 \%$ and $0.8 \%$ for 0 . Therefore we conclude that it is reasonable to use the simple Slater's approach while evaluating the effect of increasing atomic radius of neutral species on the transport coefficients.
In Table 3, the Slater diameters of the first 1-14 electronic energy levels for $N$ and $O$ are given. One can see a non-monotonic dependence of diameters on the electronic configuration. Moreover, for the nitrogen states $11-13$ we obtain the same collision diameters as for the ground state. For both $N$ and $O$ atoms, the diameters of four low-lying electronic states take the same value as for the ground state.

Since no simple explanation of this effect for many-electron atom can be given, the simplest analogy can be drawn using hydrogen-like atom, whose atomic radius as a function of electronic energy level increases as squared principle quantum number $n^{2}$ (see (16)). Following this, the atomic radius behaviour of hydrogen-like atomic species reflects the behaviour of $n^{2}$, where $n$ gradually increases with the number of electronic level. Similarly to this, the radius of many-electron atomic species also depends on the principle quantum number (more rigorously, on its effective

Table 3

N and O Atomic Spectra Levels $[39,40]$ and Slater Diameters Data.

\begin{tabular}{|c|c|c|c|c|c|c|}
\hline \multirow{2}{*}{$\begin{array}{l}\text { Electronic level } \\
\#\end{array}$} & \multicolumn{2}{|c|}{ Configuration (term) } & \multicolumn{2}{|c|}{ Electronic level, $\left[\mathrm{cm}^{-1}\right]$} & \multicolumn{2}{|l|}{$\sigma_{\text {Slater }}, \AA$} \\
\hline & $N$ & 0 & $N$ & 0 & $N$ & 0 \\
\hline 1. & $2 \mathrm{~s} 2.2 \mathrm{p} 3(4 \mathrm{~S})$ & $2 \mathrm{~s} 2.2 \mathrm{p} 4(3 \mathrm{P})$ & 0 & 0 & 3.15686 & 2.96303 \\
\hline 2. & $2 \mathrm{~s} 2.2 \mathrm{p} 3(2 \mathrm{D})$ & $2 \mathrm{~s} 2.2 \mathrm{p} 4(3 \mathrm{P})$ & 19224.464 & 158.265 & 3.15686 & 2.96303 \\
\hline 3. & $2 \mathrm{~s} 2.2 \mathrm{p} 3(2 \mathrm{D})$ & $2 \mathrm{~s} 2.2 \mathrm{p} 4(3 \mathrm{P})$ & 19233.177 & 226.977 & 3.15686 & 2.96303 \\
\hline 4. & $2 \mathrm{~s} 2.2 \mathrm{p} 3(2 \mathrm{P})$ & $2 \mathrm{~s} 2.2 \mathrm{p} 4(1 \mathrm{D})$ & 28838.92 & 15867.862 & 3.15686 & 2.96303 \\
\hline 5. & $2 \mathrm{~s} 2.2 \mathrm{p} 3(2 \mathrm{P})$ & $2 \mathrm{~s} 2.2 \mathrm{p} 4(1 \mathrm{~S})$ & 28839.306 & 33792.583 & 3.15686 & 2.96303 \\
\hline 6. & $2 \mathrm{~s} 2.2 \mathrm{p} 2.3 \mathrm{~s}(4 \mathrm{P})$ & $2 \mathrm{~s} 2.2 \mathrm{p} 3.3 \mathrm{~s}(5 \mathrm{~S})$ & 83284.07 & 73768.2 & 8.74545 & 8.15013 \\
\hline 7. & $2 \mathrm{~s} 2.2 \mathrm{p} 2.3 \mathrm{~s}(4 \mathrm{P})$ & $2 \mathrm{~s} 2.2 \mathrm{p} 3.3 \mathrm{~s}(3 \mathrm{~s})$ & 83317.83 & 76794.978 & 8.74545 & 8.15013 \\
\hline 8. & $2 \mathrm{~s} 2.2 \mathrm{p} 3.3 \mathrm{~s}(4 \mathrm{P})$ & $2 \mathrm{~s} 2.2 \mathrm{p} 3.3 \mathrm{p}(5 \mathrm{P})$ & 83364.62 & 86625.757 & 8.74545 & 8.15013 \\
\hline 9. & $2 \mathrm{~s} 2.2 \mathrm{p} 3.3 \mathrm{~s}(2 \mathrm{P})$ & $2 \mathrm{~s} 2.2 \mathrm{p} 3.3 \mathrm{p}(5 \mathrm{P})$ & 86137.35 & 86627.778 & 8.74545 & 8.15013 \\
\hline 10. & $2 \mathrm{~s} 2.2 \mathrm{p} 3.3 \mathrm{~s}(2 \mathrm{P})$ & $2 \mathrm{~s} 2.2 \mathrm{p} 3.3 \mathrm{p}(5 \mathrm{P})$ & 86220.51 & 86631.454 & 8.74545 & 8.15013 \\
\hline 11. & $2 \mathrm{~s} .2 \mathrm{p} 4(4 \mathrm{P})$ & $2 \mathrm{~s} 2.2 \mathrm{p} 3.3 \mathrm{p}(3 \mathrm{P})$ & 88107.26 & 88630.587 & 3.15686 & 8.15013 \\
\hline 12. & $2 \mathrm{~s} .2 \mathrm{p} 4(4 \mathrm{P})$ & $2 \mathrm{~s} 2.2 \mathrm{p} 3.3 \mathrm{p}(3 \mathrm{P})$ & 88151.17 & 88631.146 & 3.15686 & 8.15013 \\
\hline 13. & $2 \mathrm{~s} .2 \mathrm{p} 4(4 \mathrm{P})$ & $2 \mathrm{~s} 2.2 \mathrm{p} 3.3 \mathrm{p}(3 \mathrm{P})$ & 88170.57 & 88631.303 & 3.15686 & 8.15013 \\
\hline 14. & $2 \mathrm{~s} 2.2 \mathrm{p} 2.3 \mathrm{p}(2 \mathrm{P})$ & $2 \mathrm{~s} 2.2 \mathrm{p} 3.4 \mathrm{~s}(5 \mathrm{~S})$ & 93581.55 & 95476.728 & 8.74545 & 18.24683 \\
\hline
\end{tabular}


value $n^{*}$, see (18)), the dependence is however linear. In addition to this, the behaviour of principle quantum number $n$ of many-electron atoms is non-monotonic [39]. Thus, as a rough approximation, the behaviour of atomic radius of an electronically excited manyelectron atom corresponds to the non-monotonic behaviour of effective quantum number $n^{*}$.

In Fig. 2, the radius of neutral atoms as a function of electronic energy and the Slater diameter as a function of electronic level are presented. For atomic oxygen $O$, the excited atom radius increases faster compared to $N$. For electronic levels lower than 25 (with energy less than $12 \mathrm{eV}$ ), the radius and the corresponding Slater diameter of $\mathrm{N}$ is about three times higher than for the ground state. Contrarily to this, for the 14 th level of $O, \sigma$ related to the Slater diameter for the ground electronic state is about 6 (see Fig.2), and after some fluctuations in the middle-lying levels $14-50$, it shows further increasing. For $N$, the diameter variation for highlying states is weak: for levels $50-160$ the diameter is close to $6 \sigma_{11}\left(\sigma_{n m}\right.$ represents the effective cross-section for the collision of two electronically excited species on levels $n$ and $m$ correspondingly). This can be attributed to the behaviour of the principle quantum number which determines the radius. Thus for oxygen, the principle quantum number for the states above 150 increases and attains 24 whereas for nitrogen it is approximately 6 for all high-lying states. This also explains the fact that collision diameters for highly located states in nitrogen are significantly lower compared to the diameters of corresponding states in oxygen (see Fig. 2). The physical reason for this is associated to the form of electron configuration in the atomic shell: while for $N(1 s 2.2 s 2.2 p 3)$ the electron is removed from a half-filled $2 \mathrm{p}$ orbital, for $O(1 s 2.2 s 2.2 p 4)$ two electrons in $2 \mathrm{px}$-orbitals are coupled to each other but one of them can be easier removed since the resulting 2 p3 configuration has a low energy [25]. Therefore the electron, which has to be removed to ionize $O$, has a lower electron-nuclear attraction force than for the same electron of $N$; consequently, the atomic radius of $O$ increases faster with electronic level.

It is emphasized in [32] that for ionized mixture flows the factor of lowering the ionization energy due to the presence of charged particles has to be taken into account. In the present study we use the criteria suggested in [41]: $\varepsilon_{n}^{c} \leqslant E_{c}-\Delta E_{c}, E_{c}$ is the ionization energy, and the above mentioned lowering factor $\Delta E_{c}$ is set to $1000 \mathrm{~cm}^{-1}$. The value of $\Delta E_{c}=1000 \mathrm{~cm}^{-1}$ is chosen based on the good agreement of our results for the specific heats with those reported in [41] for the given number of accounted electronic energy of each species in the mixture; the relative error for the specific heats is within $5 \%$. Also this value for $\Delta E_{c}$ allows us to cut-off the very high-lying electronic states, that are usually unpopulated in real flows. The above assumption yields the following numbers of electronic levels and corresponding energies: $L_{N}=170$ and $\varepsilon_{N_{170}}=114,027 \mathrm{~cm}^{-1} ; \quad L_{N^{+}}=157$ $\varepsilon_{N_{157}^{+}}=230,832 \mathrm{~cm}^{-1} ; L_{O}=204, \varepsilon_{O_{204}}=109,647 \mathrm{~cm}^{-1} ; L_{O^{+}}=254$, $\varepsilon_{O_{254}^{+}}^{+}=282,219 \mathrm{~cm}^{-1}$.

It is clearly seen (see Figs. 2 and 3), that the number of accounted electronic energy levels chosen in the present study satisfies not only the Debye-Huckel criteria, but also the confined atom approximation model for the initial conditions of Fire II and Hermes (see Fig. 1). Moreover, for ionized mixtures under atmospheric pressure, the Debye-Huckel criteria also can be implemented. The only case when the increasing radius of the excited atom should be taken with care corresponds to the confined atom approximation model for the atmospheric pressure and temperature $T>10000 \mathrm{~K}$. It is obvious that under this condition, ionization is of importance and the confined atom approximation model represents only a rough approximation to the Debye-Huckel criteria. Thus we can conclude that for the atmospheric entry conditions studied in the present paper, the atomic radii calculated using the Slater's approach are in line with the results obtained for the maximum atomic radius $r_{\max }$ allowed by the Debye-Huckel criteria.

\subsection{Effective collision cross-section for resonant ion-parent atom collisions}

Along with neutral-neutral atomic collisions one of the most important processes in plasma diffusion and energy transfer is the resonant charge-transfer process during collisions of an ion and a parent atom [32,11]. This type of collisions influences the diffusion-type collision integrals (i.e.the odd-order $\Omega_{c_{n} d_{m}}^{(k, r)}, k=o d d$ ) and is characterized by very large cross-sections. For this reason, these collisions can not be neglected in the calculation of transport properties of thermal plasma flows [11]. The process of charge transfer has a high probability at large separation between colliding particles and can be represented as follow:
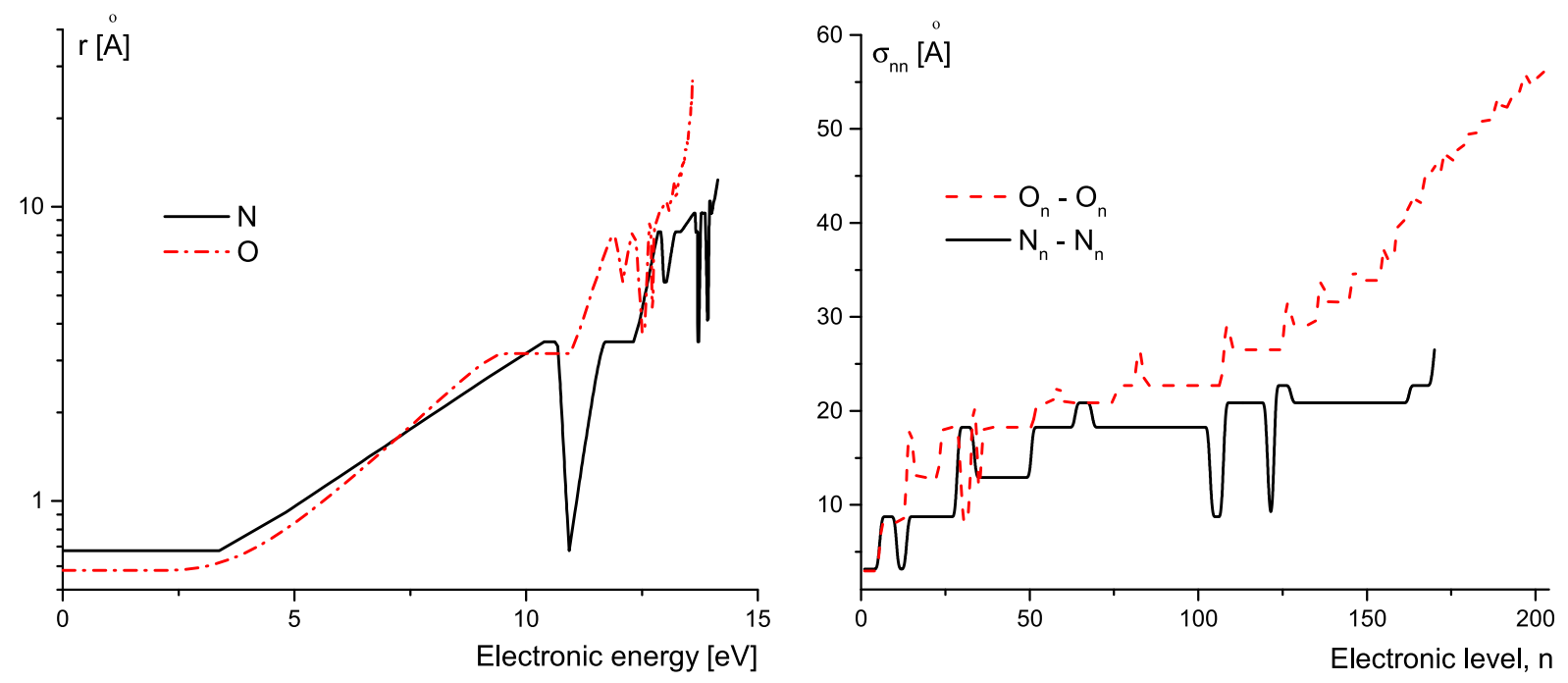

Fig. 2. Atomic radius and effective collision cross-section $\sigma_{n n}$ as a function of electronic energy and electronic level for neutral atoms. 

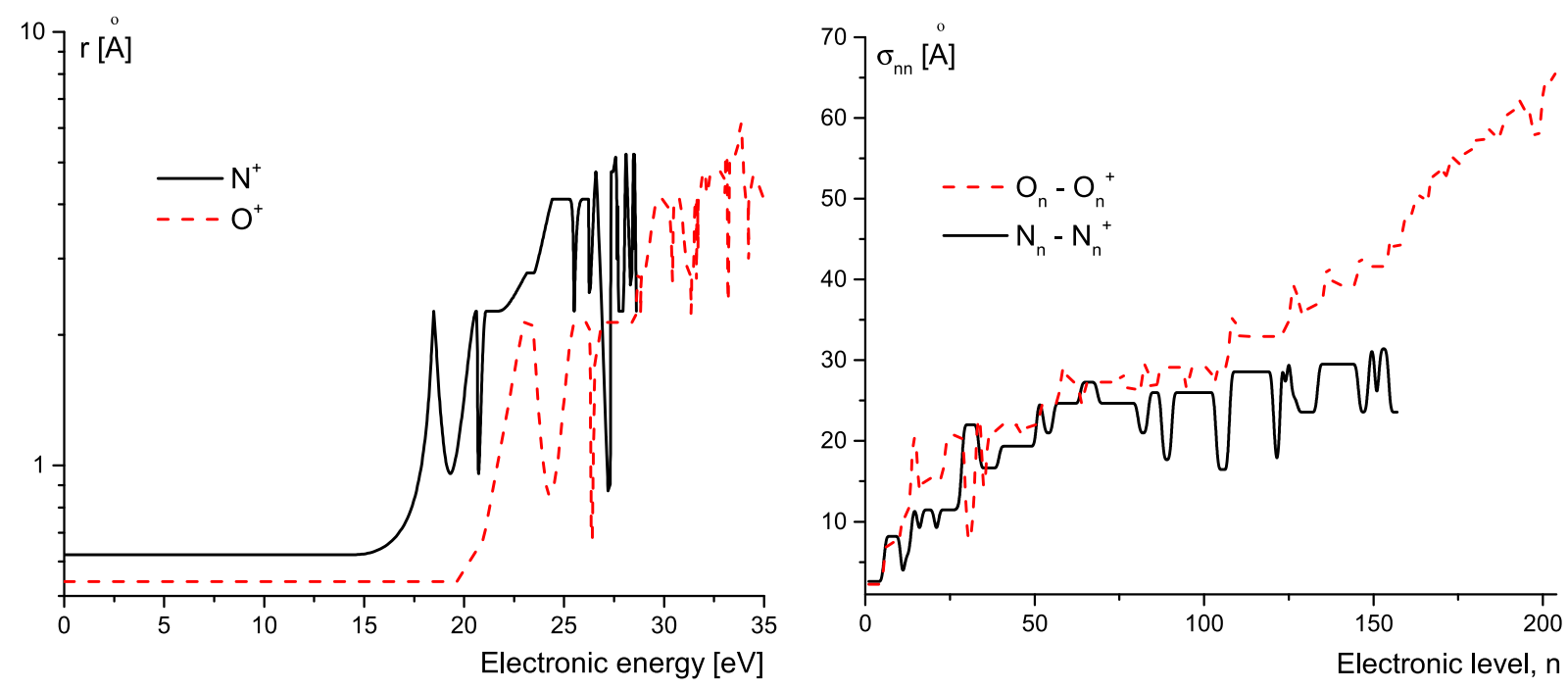

Fig. 3. Atomic radius and effective collision cross-section $\sigma_{n n}$ for ionized atomic species as a function of electronic energy and electronic level.

$X_{n}+X_{m}^{+} \Rightarrow\left(X_{2}^{+} \Rightarrow\right) X_{n}^{+}+X_{m}, \quad X=N, O$

i.e. through formation of quasi-molecular ion of X-species. When the system is transformed in a basis of states so that the interaction is localized within a small region of distances, the interaction is restricted to two states strongly coupled in a localized non-adiabatic region, the so-called two state approximation, and the resonant charge-exchange process can occur through the interaction of two distinct pathways owing to the symmetry of the quasi-molecule states [11]. At large distances the molecular arrangements of $X_{m} X_{n}^{+}$and $X_{n}^{+} X_{m}$ degenerate, and exchange interaction splits the molecular states into a pair of symmetric gerade and antisymmetric ungerade terms, with respect to the exchange of nuclei [11].

There are two widely used approaches for estimation of chargetransfer cross-sections. The most rigourous quantum approach solves the Schrödinger equations for the wave function, that makes possible to represent the total scattering cross-section as a sum of the cross-sections due to: (1) direct elastic scattering; (2) charge exchange; and (3) the term due to the interference (achieves minimal value when the classical limit is attained) [11]. In colliding systems involving open-shell atoms, the resonant charge-exchange process usually occurs through more than one pair of geradeungerade states and the relevant cross-section can be obtained by averaging the partial contributions with suitable statistical weights [11].

Another (asymptotic) approach for estimation of resonant charge-transfer cross-sections is based on the idea of expanding the cross-section in terms of small parameter connected to the geometry of the collision $[3,11]$. This approach gives the form of transport cross-section as a sum of doubled effective cross-section and so-called polarization correction, which is usually neglected for high-temperature flows [11]. With this in mind, effective odd-order collision integrals are usually considered on the basis of the classical-trajectory formulation resulting in the geometrical mean of elastic and inelastic resonant contributions [11]:

$\Omega^{(l, s) *}=\sqrt{\left(\Omega_{e l}^{(l, s) *}\right)^{2}+\left(\Omega_{c h-t r}^{(l, s) *}\right)^{2}}, \quad l=$ odd,

in which the transport cross-section has the following form:

$\sigma=2 \sigma_{e f f}=2\left(r_{i}+r_{j}\right), \quad r_{i}=\frac{2 n_{i}^{*}+1}{2 \varsigma} a_{0}$,

and atomic radii $r_{i}$ and $r_{j}$ can be found using the Slater formula.

Atomic radii of different species are presented in Table 4. While $r_{1}$ is calculated using the Slater's approach, $r_{2}$ is obtained through the backward transformation from $\sigma_{4}$ (the data in [24]) to the atomic radius. Comparison shows that for atomic nitrogen, using the Slater's approach yields slightly overestimated atomic radius, whereas for atomic oxygen the radius is underestimated. The discrepancy varies from $0.76 \%$ for $N$ up to $5.24 \%$ for $N^{+}$. Nevertheless for the effective resonant ion-atom cross-section this discrepancy is even lower and does not exceed $2.9 \%$ (see Table 2). Thus we can conclude that applying the Slater's approach yields a satisfactory accuracy of the effective cross-Section (25) for the resonant charge-transfer process.

Let us discuss now the variation of ionized species radius with the electronic energy. In Table 5 the atomic radii of the first 1-14 electronic energy levels for $\mathrm{N}^{+}$and $\mathrm{O}^{+}$are shown. One can notice a considerable increase in $N^{+}$and $O^{+}$radius from 12th and 11th levels correspondingly. Nevertheless, this increase is lower than that for neutral atomic species.

While for neutral atomic species the atomic radius increases with the energy (especially for oxygen, see Fig. 2), for ionized species starting from the energy about $25 \mathrm{eV}$ this increase slows down and $r_{n}$ fluctuates near the constant value of $4 \AA$ (see Fig. 3). Thus according to (25) the increase in charge-transfer collision diameter

Table 4

Atomic radius of neutral and ionized nitrogen and oxygen at the ground state calculated with different models.

\begin{tabular}{|c|c|c|c|c|}
\hline Atom & Configuration (term) & $\left.r_{1},[\AA]\right]$ & $\left.r_{2},[\AA]\right]$ & $\frac{r_{1}-r_{2}}{r_{2}} \cdot 100 \%[\%]$ \\
\hline$N$ & $2 \mathrm{~s} 2.2 \mathrm{p} 3(4 \mathrm{~S})$ & 0.67843 & 0.67331 & 0.76 \\
\hline 0 & $2 \mathrm{~s} 2.2 \mathrm{p} 4(3 \mathrm{P})$ & 0.58152 & 0.60624 & -4.08 \\
\hline$N^{+}$ & $2 \mathrm{~s} 2.2 \mathrm{p} 2(3 \mathrm{P})$ & 0.62256 & 0.59155 & 5.24 \\
\hline $\mathrm{O}^{+}$ & $2 \mathrm{~s} 2.2 \mathrm{p} 3(4 \mathrm{~S})$ & 0.53997 & 0.54623 & -1.15 \\
\hline
\end{tabular}


Table 5

$\mathrm{N}^{+}$and $\mathrm{O}^{+}$Atomic Spectra Levels $[39,40]$ and atomic radius, obtained using Slater's approach.

\begin{tabular}{|c|c|c|c|c|c|c|}
\hline \multirow{2}{*}{$\begin{array}{l}\text { Electronic level } \\
\#\end{array}$} & \multicolumn{2}{|c|}{ Configuration (term) } & \multicolumn{2}{|c|}{ Electronic level, $\left[\mathrm{cm}^{-1}\right]$} & \multicolumn{2}{|l|}{$r, \AA$} \\
\hline & $N^{+}$ & $O^{+}$ & $N^{+}$ & $0^{+}$ & $N^{+}$ & $\mathrm{O}^{+}$ \\
\hline 1. & $2 \mathrm{~s} 2.2 \mathrm{p} 2(3 \mathrm{P})$ & $2 \mathrm{~s} 2.2 \mathrm{p} 3(4 \mathrm{~S})$ & 0 & 0 & 0.62256 & 0.53977 \\
\hline 2. & $2 \mathrm{~s} 2.2 \mathrm{p} 2(3 \mathrm{P})$ & $2 \mathrm{~s} 2.2 \mathrm{p} 3(2 \mathrm{D})$ & 48.7 & 26810.55 & 0.62256 & 0.53977 \\
\hline 3. & $2 \mathrm{~s} 2.2 \mathrm{p} 2(3 \mathrm{P})$ & $2 \mathrm{~s} 2.2 \mathrm{p} 3(2 \mathrm{D})$ & 130.8 & 26830.57 & 0.62256 & 0.53977 \\
\hline 4. & $2 \mathrm{~s} .2 \mathrm{p} 3(5 \mathrm{~S})$ & $2 \mathrm{~s} 2.2 \mathrm{p} 3(2 \mathrm{P})$ & 46784.6 & 40468.01 & 0.62256 & 0.53977 \\
\hline 5. & $2 \mathrm{~s} .2 \mathrm{p} 3(3 \mathrm{D})$ & $2 \mathrm{~s} 2.2 \mathrm{p} 3(2 \mathrm{P})$ & 92237.2 & 40470.00 & 0.62256 & 0.53977 \\
\hline 6. & $2 \mathrm{~s} .2 \mathrm{p} 3(3 \mathrm{D})$ & $2 \mathrm{~s} .2 \mathrm{p} 4(4 \mathrm{P})$ & 92250.3 & 119837.21 & 0.62256 & 0.53977 \\
\hline 7. & $2 \mathrm{~s} .2 \mathrm{p} 3(3 \mathrm{D})$ & $2 \mathrm{~s} .2 \mathrm{p} 4(4 \mathrm{P})$ & 92251.8 & 120000.43 & 0.62256 & 0.53977 \\
\hline 8. & $2 \mathrm{~s} .2 \mathrm{p} 3(3 \mathrm{P})$ & $2 \mathrm{~s} .2 \mathrm{p} 4(4 \mathrm{P})$ & 109216.6 & 120082.86 & 0.62256 & 0.53977 \\
\hline 9. & $2 \mathrm{~s} .2 \mathrm{p} 3(3 \mathrm{P})$ & $2 \mathrm{~s} .2 \mathrm{p} 4(2 \mathrm{D})$ & 109217.6 & 165988.46 & 0.62256 & 0.53977 \\
\hline 10. & $2 \mathrm{~s} .2 \mathrm{p} 3(3 \mathrm{P})$ & $2 \mathrm{~s} .2 \mathrm{p} 4(2 \mathrm{D})$ & 109223.5 & 165996.50 & 0.62256 & 0.53977 \\
\hline 11. & $2 \mathrm{~s} .2 \mathrm{p} 3(1 \mathrm{D})$ & $2 \mathrm{~s} 2.2 \mathrm{p} 2.3 \mathrm{~s}(4 \mathrm{P})$ & 144187.94 & 185235.281 & 0.62256 & 2.13706 \\
\hline 12. & $2 \mathrm{~s} 2.2 \mathrm{p} .3 \mathrm{~s}(3 \mathrm{P})$ & $2 \mathrm{~s} 2.2 \mathrm{p} 2.3 \mathrm{~s}(4 \mathrm{P})$ & 148908.59 & 185340.577 & 2.2679 & 2.13706 \\
\hline 13. & $2 \mathrm{~s} 2.2 \mathrm{p} .3 \mathrm{~s}(3 \mathrm{P})$ & $2 \mathrm{~s} 2.2 \mathrm{p} 2.3 \mathrm{~s}(4 \mathrm{P})$ & 148940.17 & 185499.124 & 2.2679 & 2.13706 \\
\hline 14. & $2 \mathrm{~s} 2.2 \mathrm{p} .3 \mathrm{~s}(3 \mathrm{P})$ & $2 \mathrm{~s} 2.2 \mathrm{p} 2.3 \mathrm{~s}(2 \mathrm{P})$ & 149076.52 & 188888.543 & 2.2679 & 2.13706 \\
\hline
\end{tabular}

for high electronic states is mainly due to increasing of the atomic radius of neutral species. At the same time, an opposite effect is observed for the effective cross-sections of neutral-neutral and resonant neutral-ionized collisions: effective cross-sections for collisions of neutral species remain nearly constant for 12-150 electronic energy levels (about 6 times larger for high-energy levels compared to that for the ground electronic state), whereas for resonant charge-transfer collisions the effective cross-section for high-energy levels is greater for both $\mathrm{N}^{+}$and $\mathrm{O}^{+}$, and, moreover, keeps growing for $\mathrm{O}^{+}$. Thus we can conclude that for high energy levels the ratio $\sigma_{n n} / \sigma_{11}$ for resonant charge-exchange collisions $\left(X-X^{+}\right)$is approximately two times higher than the corresponding cross-section for neutral species collisions $(X-X)$, as was predicted by (25).

\section{Distribution of electronically excited states behind the shock front}

Since considerable increase in effective cross-sections leads to high values of collision integrals, non-equilibrium distributions over excited electronic levels may play an important role in calculation of transport properties. Let us consider a strongly non-equilibrium flow behind the shock wave under Fire II conditions. This experiment represents an excellent example of reentry from interplanetary flight mission, that is why the case of Fire II has been staying in the focus of many researchers and remains widely discussed in the literature for the last fifty years $[23,21,22,42,33,12]$.

In Refs. [21,22,42], a general mechanism of electronic excitation of atomic species in electron-impact reactions is proposed. In these studies a collisional radiative model developed in [43-45,21] is implemented, and for the case of Fire II all relevant collisional and radiative processes are taken into account $[21,22,42]$. For accurate estimates of ionization and population of excited states (including metastable) that are easily induced by electrons due to their small mass and high velocity, the following numbers of electronic energy levels are accounted: 46 for $N$, and 40 for $O$ $[21,22,42]$. For electronic transitions the rate coefficients represent a function of the secondary quantum number $l$ of each level for optically allowed and forbidden transitions [21,22,42]. Thorough analysis of obtained numerical results shows that behind the shock front the distribution of atomic nitrogen and oxygen over electronic states becomes Boltzmannian at the distance of about $2.5 \mathrm{~cm}$ and further, while within the distance $x=0-1 \mathrm{~cm}$, the distribution over electronic states represents a combination of the Boltzmann distribution for low levels:

$n_{n}=n_{a t} \frac{g_{n} \exp \left(-\frac{\varepsilon_{n}}{k T}\right)}{Z_{\text {int }}}$, and the Saha distribution modified for electronic excitation [21,22] for high levels:

$n_{n}=n_{\text {ion }} n_{e}\left(\frac{h^{2}}{2 m_{e} \pi k T}\right)^{3 / 2} \frac{g_{n} \exp \left(-\frac{\varepsilon_{n}-\varepsilon_{\text {ion }}}{k T}\right)}{Z_{\text {int }}^{\text {ion }}}$.

Here $\varepsilon_{n}$ and $\varepsilon_{i o n}$ are respectively the energies of the electronic state $n$ and ionization energy of atomic species, $n_{a t}, n_{\text {ion }}$ and $n_{e}$ are number densities of neutral and ionized atomic species and that of electrons, $g_{n}$ is the statistical weight for the electronic state $n, Z_{\text {int }}(T)$ is the equilibrium internal partition function. These two distributions are combined into the third one (further in the paper it is called the combined distribution), for which low-lying electronic energy levels are highly populated whereas the population of high-lying levels is greater than corresponding values for the modified Saha distribution, but remains lower than values for the Boltzmann distribution (this distribution represents an approximation of the results reported in [22]).

In the following section we evaluate the impact of electronic excitation on the transport properties taking into account these two kinds of distributions, which can be observed behind the shock front: the combined state-to-state distribution representing a superposition of the Boltzmann and Saha ones taken at the specific point $x=0.7 \mathrm{~cm}(T=12,000 \mathrm{~K}, p=4200 \mathrm{~Pa})$ behind the shock wave [21,22], and the commonly used Boltzmann distribution (see Fig. 4) calculated at $x=2.5 \mathrm{~cm}(T=9000 \mathrm{~K}, p=4200 \mathrm{~Pa})$. For $N$ and $O$ we adopt this kind of distribution, whereas for ionized $\mathrm{N}^{+}$and $\mathrm{O}^{+}$there is no data for distributions over electronic states behind the shock front. Therefore due to the minor concentration of ionized species compared to that of the neutral ones we implement only the Boltzmann distribution for ions. This assumption about the Boltzmann distribution for ions makes sense for plasma applications when highly located electronic states are significantly populated. In Fig. 4 different distributions over electronic energy for $\mathrm{N}$ and $\mathrm{O}$ atoms are compared. It is seen that at $x=0.7 \mathrm{~cm}$ low-lying electronic states are dominating while the population of high-lying states is much lower and tends to the modified Saha distribution. Similar under-population of high vibrational levels in shock heated flows is reported in many papers devoted to the state-to-state vibrational kinetics (see $[16,46]$ ).

\section{Results and discussions}

In this section we evaluate the influence of electronic excitation, the size of excited species, and distributions over electronic energy in pure and ionized atomic gases on the state-resolved transport properties. Since no data are found in the literature for the collision integrals $\Omega_{c_{n} d_{m}}^{(l, r)}$ of high-lying electronic states $n$ and $m$, we propose 

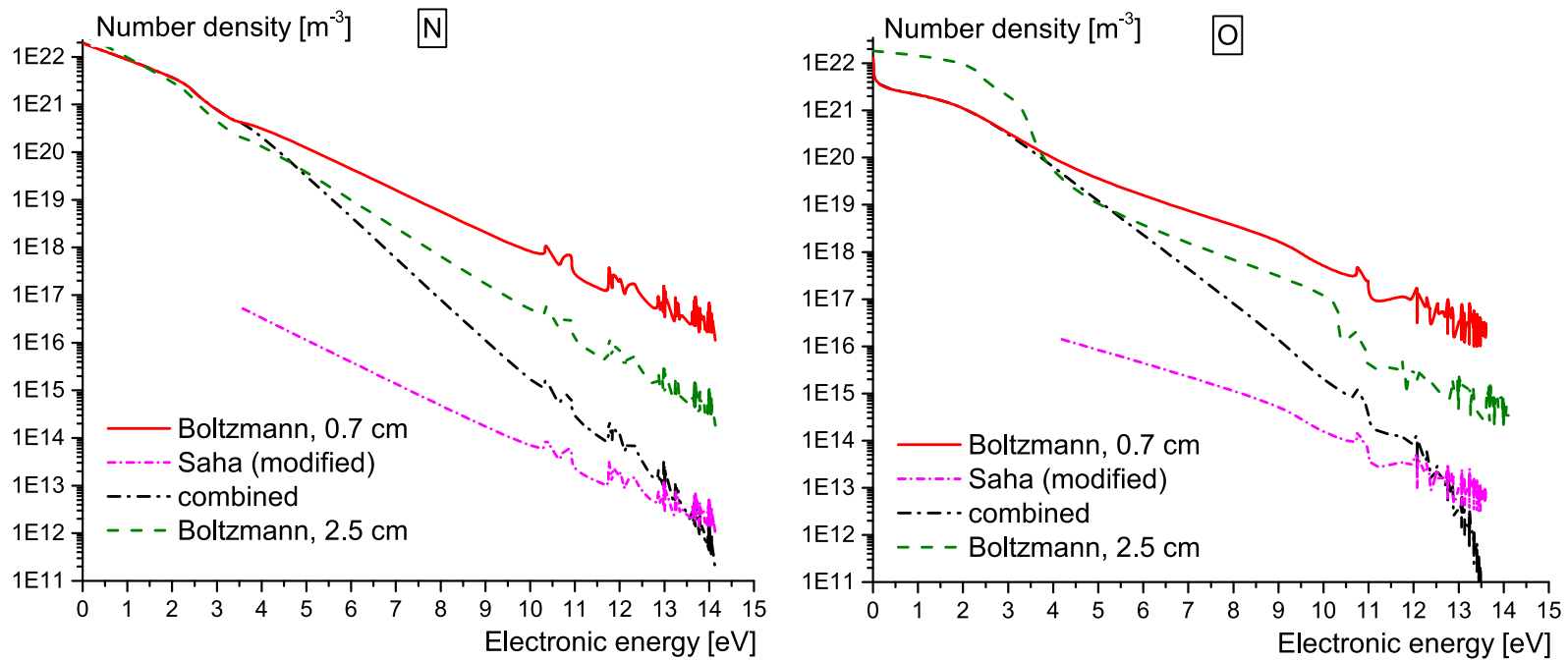

Fig. 4. Population of $N$ and $O$ electronic energy levels at $x=0.7 \mathrm{~cm}$ and $2.5 \mathrm{~cm}$ behind the shock wave for two distributions.

to evaluate them using the Slater's approach for the calculation of atomic radius of excited species and effective collision diameter for neutral-neutral and ion-neutral collisions. The effect of the number of accounted electronic levels on the transport terms is also estimated.

\subsection{Thermal conductivity coefficient}

In Figs. 5 and 6 the partial thermal conductivity coefficient $\lambda^{\prime}$ of pure atomic $N$ and $O$, and ionized $N / N^{+} / e^{-}$and $O / O^{+} / e^{-}$mixtures is shown as a function of $T$. The coefficients are plotted for the following particular cases:

1. Atomic species at the ground electronic states (the ground-state model). It is worth noting that under this assumption thermal conductivity coefficient $\lambda^{\prime}$ corresponds to the translational thermal conductivity coefficient $\lambda_{\text {tr }}$ in the one-temperature approach [16], without taking into account electronic excitation.

2. Combined distribution at $x=0.7 \mathrm{~cm}$ (for fixed $T=12,000 \mathrm{~K}$ ) considered with the following number of accounted electronic energy levels: 170 for $N, 157$ for $N^{+}, 204$ for 0,254 for $O^{+}$. It means that while using the combined distribution we take into account all electronic states of all species. We should remind that for this particular case, ionized atomic species are distributed accordingly to the Boltzmann distribution with the temperature $T$, whereas neutral species follow the combination of the Boltzmann and Saha distributions.

3. The Boltzmann distribution as a function of temperature $T$ :

(a) for pure atomic gases, the thermal conductivity coefficient $\lambda^{\prime}$ is calculated with $6,13,50$ and 170 (204) electronic states;

(b) for ionized mixtures, the thermal conductivity coefficient $\lambda^{\prime}$ as a function of $T$ is calculated for both neutral atoms (50 and 170 for $N$, and 50 and 204 for 0 ) and ionized atomic species (50 and 157 for $N^{+}$, and 50 and 254 for $\mathrm{O}^{+}$).

First we discuss the results for pure atomic gases with electronic excitation (see Fig. 5). For the temperature range 1000-14,000 K all the results coincide, because just behind the shock front the distribution over electronic energy is determined mainly by the lowlying states. As soon as high-lying states become populated, the thermal conductivity coefficient sharply decreases. This decrease
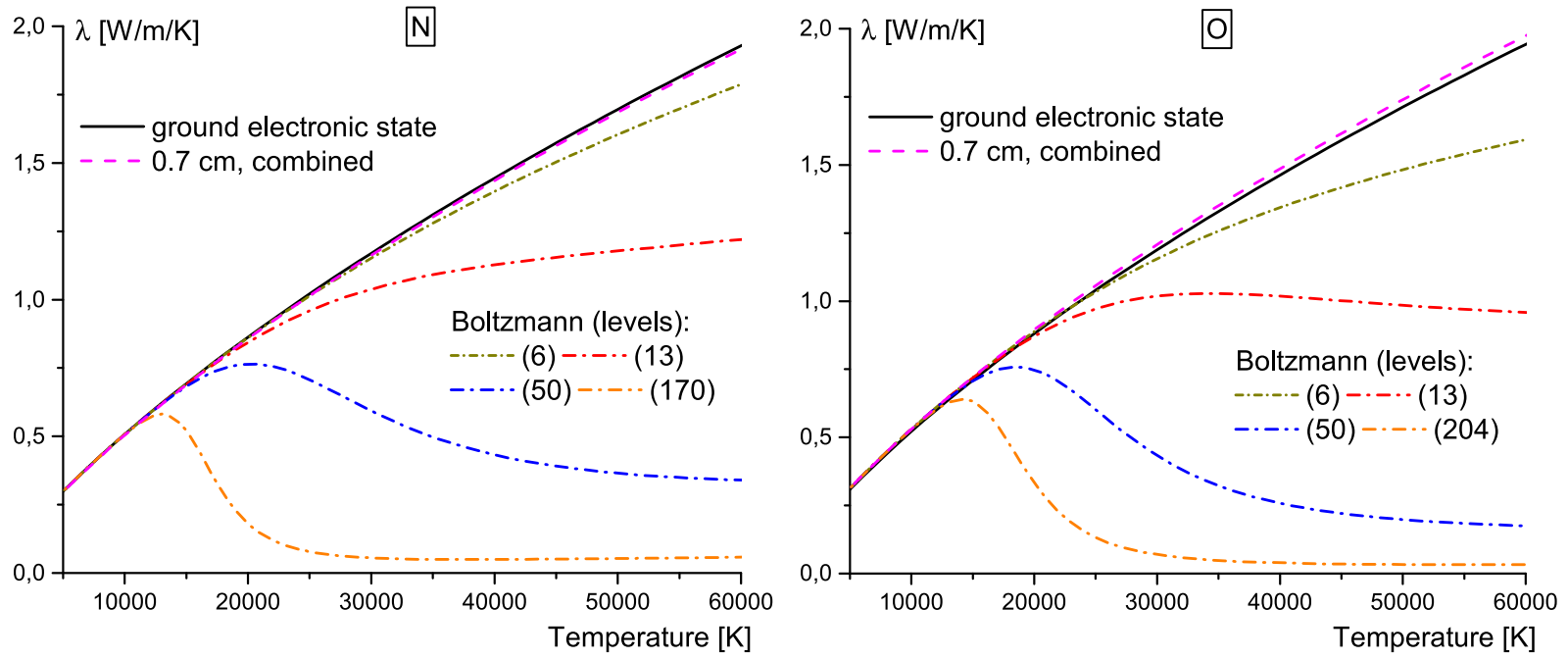

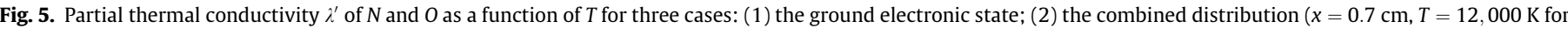
neutral atoms; the Boltzmann for ionized atoms); and (3) the Boltzmann distribution taken for different number of electronic energy levels. 

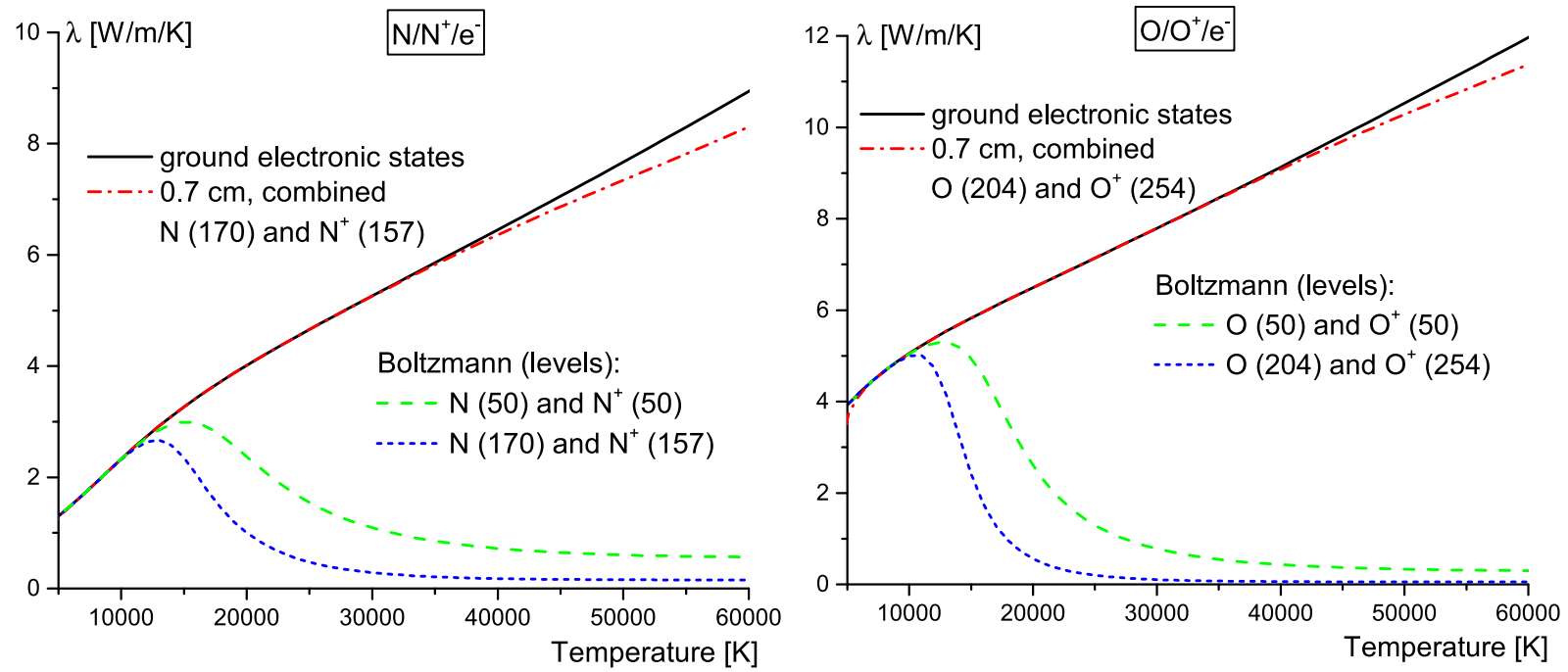

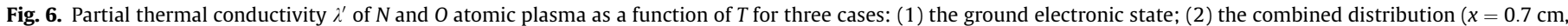
$T=12,000 \mathrm{~K}$ for neutral atoms; the Boltzmann for ionized atoms); and (3) the Boltzmann distribution taken for different number of electronic energy levels.

is governed by the number of levels taken into account since the Boltzmann distribution strongly depends on the accounted levels. This result is in line with those reported in [11]. Comparing the results for $N$ and $O$ we should keep in mind that with the rise of principal quantum number for $O$ the collision diameter becomes higher than for $N$ (see Fig. 3 ). Due to this fact, $\lambda^{\prime}$ in oxygen decreases faster compared to that in nitrogen. For the combined distribution, the thermal conductivity coefficient $\lambda^{\prime}$ nearly coincides with that for the ground electronic state and even becomes a little bit greater for 0 . This can be explained by the fact that the data [24] provides the diameter of the ground electronic state about $1.64 \%$ higher than the Slater's one (see Table 3). Since we use the data [24] for the ground electronic state and the Slater's approach otherwise, the size of the next four low-lying states becomes $1.64 \%$ less than that of the ground one (from $\sigma=3.01248$ to 2.96303 [ $\AA$ ], see Table 3). In the case of combined distributions, the molar fractions of atomic species at the ground state and at the next four low-lying levels are related roughly as 50/50\%, and this discrepancy starts to influence thermal conductivity, slightly increasing it.

Let us consider now the thermal conductivity coefficient in ionized mixtures (see Fig. 6). It is worth mentioning that for significantly ionized mixtures (the level of ionization of $5 \%$ corresponds to the case of Fire II, $1634 \mathrm{~s}, 0.7 \mathrm{~cm}$ behind the shock front) the thermal conductivity coefficient considerably exceeds that for the case of single-component gases. This increase is caused by the charge-transfer process, and by the presence of light and fast electrons that are very effective in heat transfer (especially with the rise of temperature) in the mixture. Thus we conclude that for ionized atomic mixtures, the charge-transfer process gives a significant contribution to thermal conductivity. This result confirms the well known fact that the charge-transfer process is of importance in various plasma applications due to its great influence on the process of ions diffusion (see also SubSection 6.3 on diffusion coefficients) and energy transfer [47,3].

At the same time the increasing atomic radius does not considerably influence the thermal conductivity coefficient calculated in the state-to-state approach for realistic distributions contrarily to the case of the Boltzmann distribution over electronic energy levels. Since the molar fraction of electrons is high, and the resonant charge-transfer process starts to play an important role in the transport terms (keep in mind the second term under the square root in (24)), one could expect a greater effect of the cross-section (see Fig. 3) on the thermal conductivity. However one can see that for the Boltzmann distribution, the behaviour is exactly the same as for pure atomic gases. Moreover for the case of combined distribution the effect of resonant charge-transfer appears only for the temperature of $45,000 \mathrm{~K}$ and higher, and does not exceed $10 \%$ for $60,000 \mathrm{~K}$.

Just behind the shock front for Fire II and Hermes cases, the temperature drops fast and achieves the values in the range 5000-12,000 K (see Ref. [12]), where results for all distributions are similar. Therefore, it is not necessary to take into account increasing atomic radius for the thermal conductivity coefficient calculation at the sufficient distance from the shock front. This conclusion is quite encouraging since allows considerable simplification of the transport algorithm.

\subsection{Shear viscosity coefficient}

In Figs. 7 and 8, the shear viscosity coefficient $\eta$ of single-component nitrogen and oxygen and ionized mixtures of atomic $\mathrm{N}$ and $\mathrm{O}$ is given as a function of $T$ for the same distributions as indicated in the previous section. Contrarily to thermal conductivity coefficients, viscosity coefficients for ionized mixtures and neutral gases do not differ considerably because viscosity-type collision integrals (see (24) for $l=$ even) are independent of the charge-transfer cross sections. For pure atomic $N$ and $O$, similarly to $\lambda^{\prime}$, just behind the shock front the combined distribution over electronic states yields practically the same results as for the ground state. On the contrary, shear viscosity calculated using the Boltzmann distribution strongly depends on the accounted number of electronic energy levels. The influence of the atomic size on the shear viscosity calculated for realistic distributions is stronger compared to thermal conductivity: starting from $30,000 \mathrm{~K}, \eta$ decreases with the temperature. This fact follows from the nature of the resonant chargetransfer process: while increasing effective cross-sections influence stronger the odd-term collision integrals (24), the even (viscous) terms do not contain resonant inelastic contribution of the charge-transfer process. This means that the bracket integrals $H_{00}^{c_{n} d_{m}}$ in Eq. (11) containing the ratio of even to odd collision integrals start to decrease as fast as effective cross-sections of the charge-transfer process increase. Nevertheless, for hypersonic reentry problems the effect of state-dependent collision cross-sections on the shear viscosity remains weak since for realistic distributions its considerable decrease starts at $T>30000 \mathrm{~K}$. 

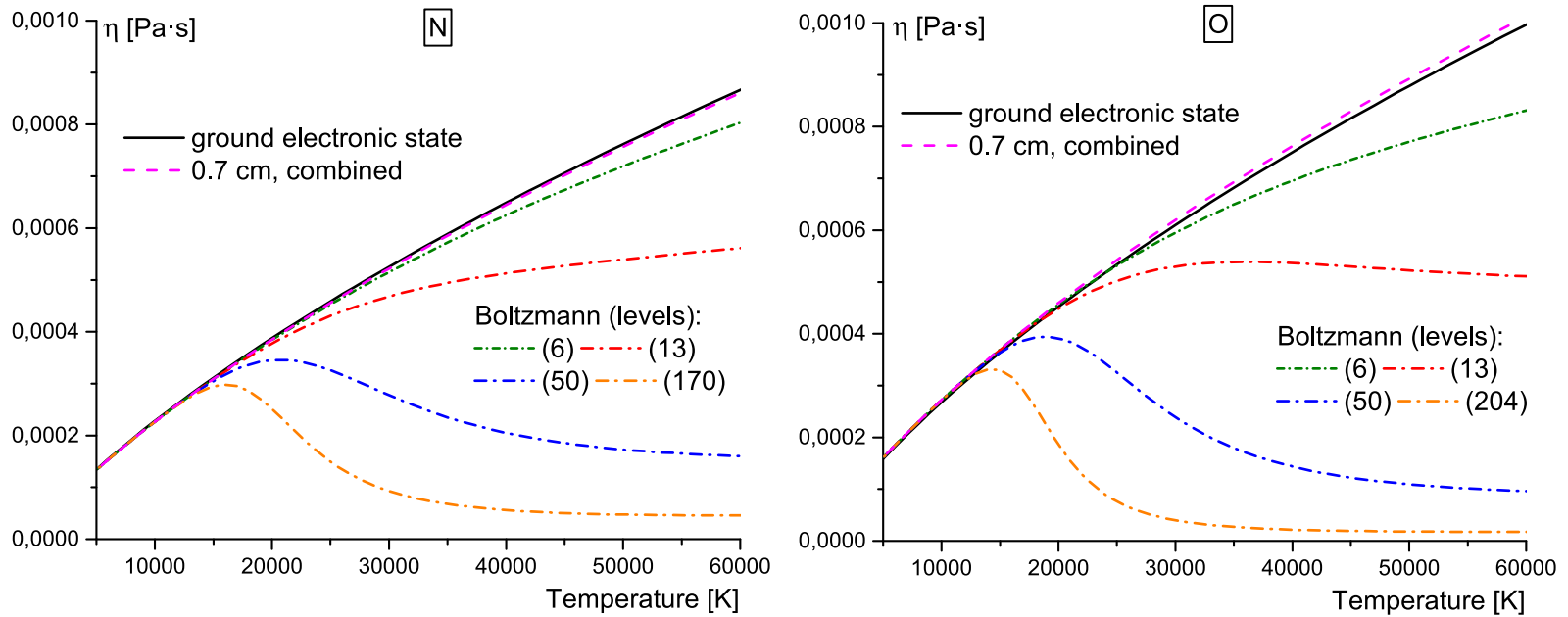

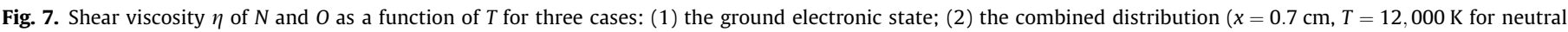
atoms; the Boltzmann for ionized atoms); and (3) the Boltzmann distribution taken for different number of electronic energy levels.
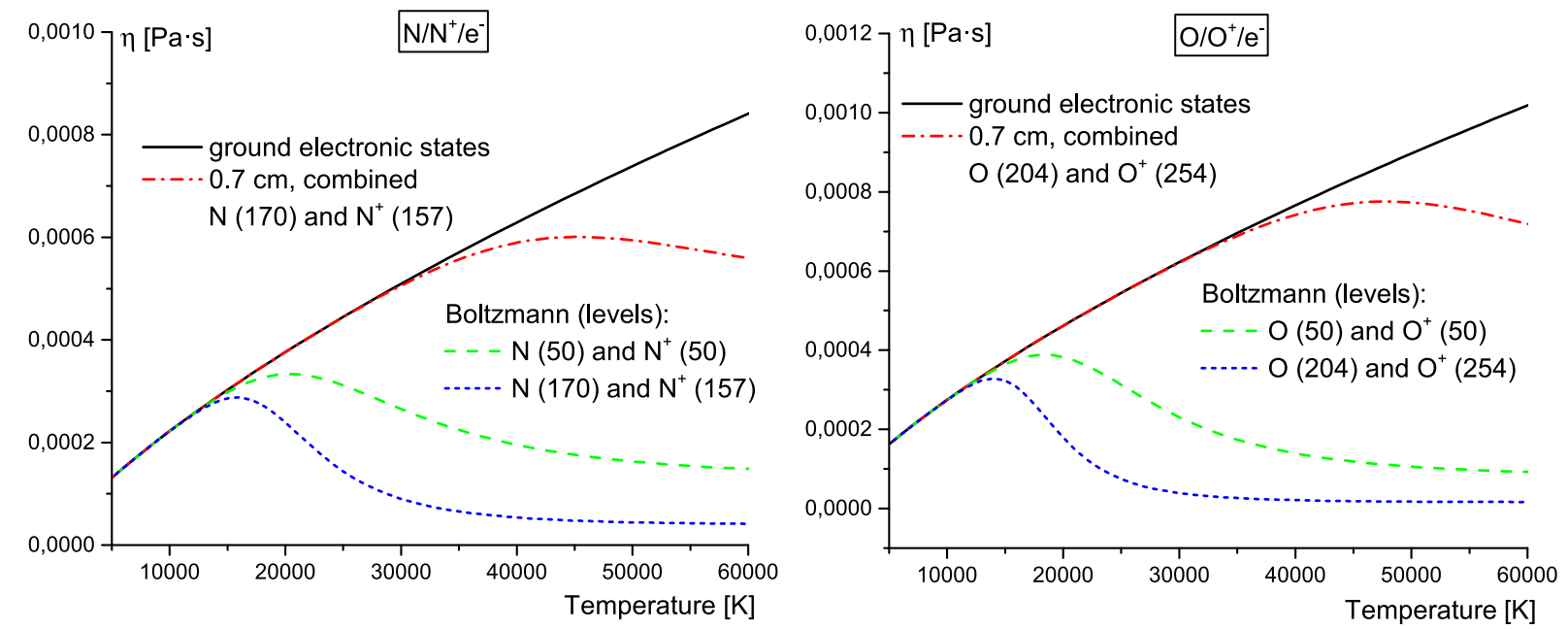

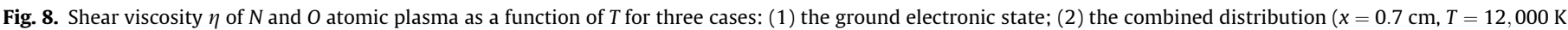
for neutral atoms; the Boltzmann for ionized atoms); and (3) the Boltzmann distribution taken for different number of electronic energy levels.

\subsection{Diffusion coefficient}

Whereas diffusion of excited vibrational species was recently studied in several papers $[48-51,46]$, investigation of diffusion and thermal diffusion of electronically excited gases, especially atomic ones, is still an open problem. Calculation of state-resolved diffusion coefficients is of importance for predictions of the heat flux (7) and diffusion velocity (6) near the surface of reentry spacecraft. This is particularly important because in the state-to-state approach, the heat flux is determined mainly by diffusion processes coupled with heat transfer due to thermal conductivity, contrarily to the one-temperature model, in which for singlecomponent gases diffusion processes do not exist $[35,16]$.

In Fig. 9, self-diffusion coefficients $D_{n n}$ of various electronic states of pure atomic nitrogen and oxygen are given at fixed $T=12,000 \mathrm{~K}$ as functions of electronic energy levels $n$ for different distributions over electronic energy levels. Three kinds of distributions are studied: the combined and Boltzmann distribution, mentioned in the previous section as well as an equiprobable distribution of atomic species over electronic energy levels. This last distribution is in some sense artificial, however, due to the fact that diffusion coefficients strongly depend on the species molar fraction (it is clearly seen in the Fig. 9), this approach can be very useful for understanding behaviour of state-resolved diffusion coefficients depending on the atomic radius. One can see that for the equiprobable distribution the diffusion coefficient slowly decreases with the electronic energy level and, consequently, atomic radius. At the same time, for the Boltzmann and combined distributions, diffusion coefficients are inversely proportional to the molar fraction, and depend on the atomic radius. As the result, for the Boltzmann distribution $D_{n n}$ is nearly constant for electronic energy levels higher than 10 , and for the combined distribution, $D_{n n}$ increase with $\mathrm{n}$ (especially for 0 ). This gives evidence of strong dependence of the diffusion coefficient not only on the molar fraction, but also on the atomic radius (i.e. effective crosssection).

Let us consider state-dependent diffusion coefficients $D_{n m}$ $(n \neq m)$ calculated on the basis of equiprobable distribution. Let us limit the number of accounted electronic energy levels to the first six levels. This is done because as is seen from Table 3, for both $\mathrm{N}$ and $\mathrm{O}$ atoms the radius is equal for $n=1 \ldots 5$ and increases starting from $n=6$. We distinguish two cases in order to understand the effect of atomic radius:

1. $r_{n}=r_{1}, \quad \forall n=1 . .6$;

2. $r_{n}=r_{1}, \quad \forall n=1 . .5 ; r_{6} \neq r_{1}$ 

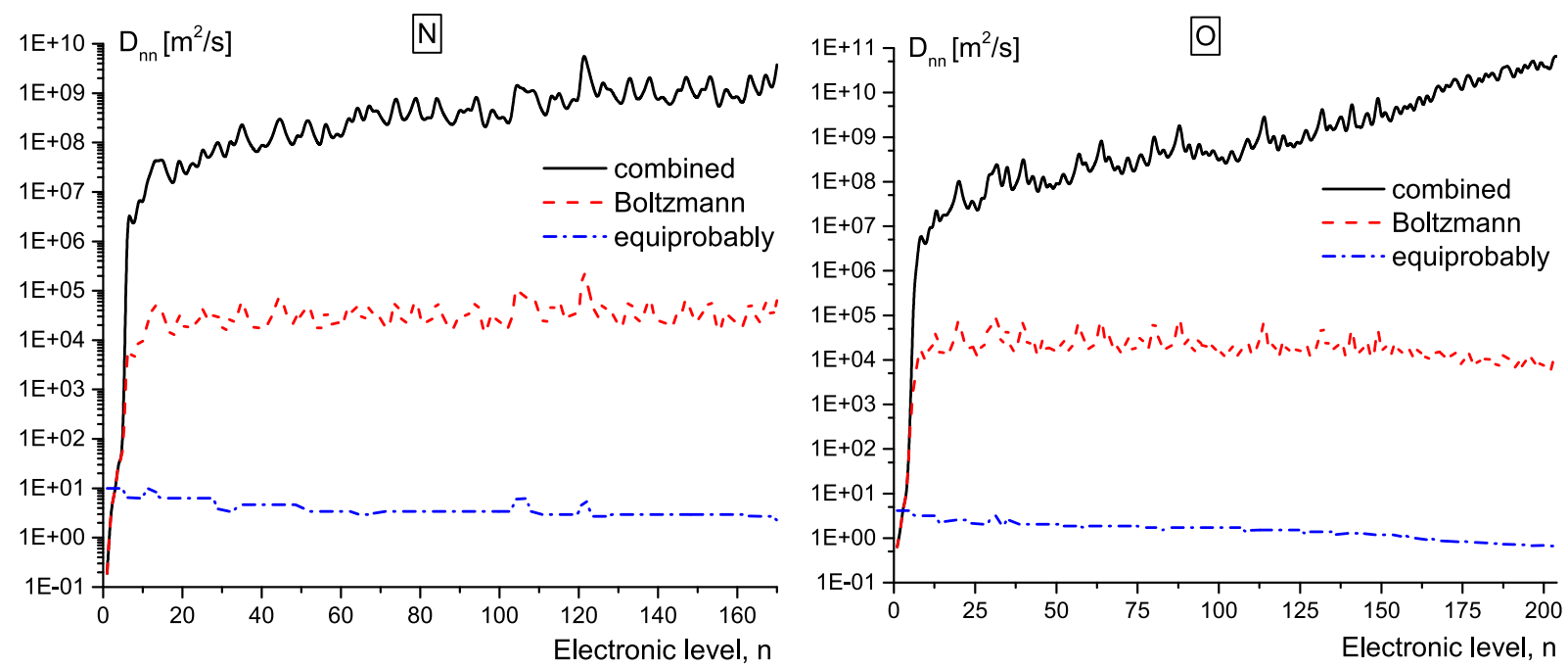

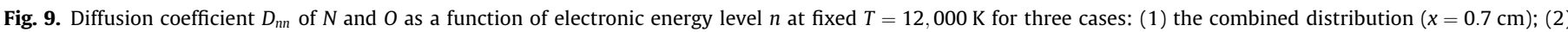
Boltzmann distribution; (3) equiprobable distribution of atoms over electronic energy levels.

For these two cases in the Fig. 10 the diffusion coefficients $D_{n m}$ are shown. Note that for the equiprobable distribution all $D_{n m}$ are equal if $r_{n}=$ const. This is not the case for the realistic distribution. Another result that might be expected from the state-tostate approach implemented for diffusion of vibrational molecular states [52,53], is that cross diffusion coefficients are equal to each other $D_{n m}=D_{m n}$ for every pair of $(n, m)$ for the first cases, but $D_{6 m} \neq D_{n m}$ if $n \neq 6$ for the second case. Increasing atomic radius of one state in a mixture of six leads to the decrease in all diffusion coefficients in pure atomic gases. This effect can be seen also from the self-diffusion coefficient $D_{n n}$, especially for $n=6$, that differs significantly from the value of $D_{n n}$ for $n=1 \ldots 5$ species. Nevertheless, this effect must be taken into account in the real flows only for the temperature range $14,000-60,000 \mathrm{~K}$, which is in line with the results for thermal conductivity and shear viscosity coefficients.

\subsection{Thermal diffusion coefficient}

In Fig. 11, the thermal diffusion coefficient $D_{T n}$ is plotted as a function of temperature $T$ for pure atomic $N$ and $O$ (6 electronic energy levels taken into account), and for ionized mixtures $\mathrm{N} / \mathrm{N}^{+} / \mathrm{e}^{-}$and $\mathrm{O} / \mathrm{O}^{+} / \mathrm{e}^{-}$(6 electronic energy levels of neutral and ionized atoms are taken into account). One has to keep in mind that in the one-temperature approach for pure atomic gases this coefficient does not appear at all [35,16]. For equiprobably distributed over first six electronic energy levels molar fractions of pure atomic gases we can see that increasing atomic radius influences significantly $D_{T n}$ : the value of $D_{T 6}$ is six times higher than corresponding absolute value for $D_{T n}, n=1 \ldots 5$ which are equal one to another since the first five levels of $\mathrm{N}$ and $\mathrm{O}$ have the same value for atomic radius (see Table 5). For ionized mixtures we plot the absolute values of thermal diffusion coefficients of ions and electrons. Since we do not account for the presence of electro-magnetic field in a plasma, the effect of ambipolar diffusion is out of consideration. As a result, large values of thermal diffusion coefficients for electrons and the corresponding heat flux can be obtained [12]. On the other hand the presence of electro-magnetic field induces the increasing effect of ambipolar diffusion which reduces the effect of thermal diffusion $[54,55]$. It is seen that thermal diffusion coefficient $D_{T_{X}}$ of neutral species is two orders of magnitude higher than $D_{T_{X^{+}}}$of ionized species $(X=N, O)$; more-
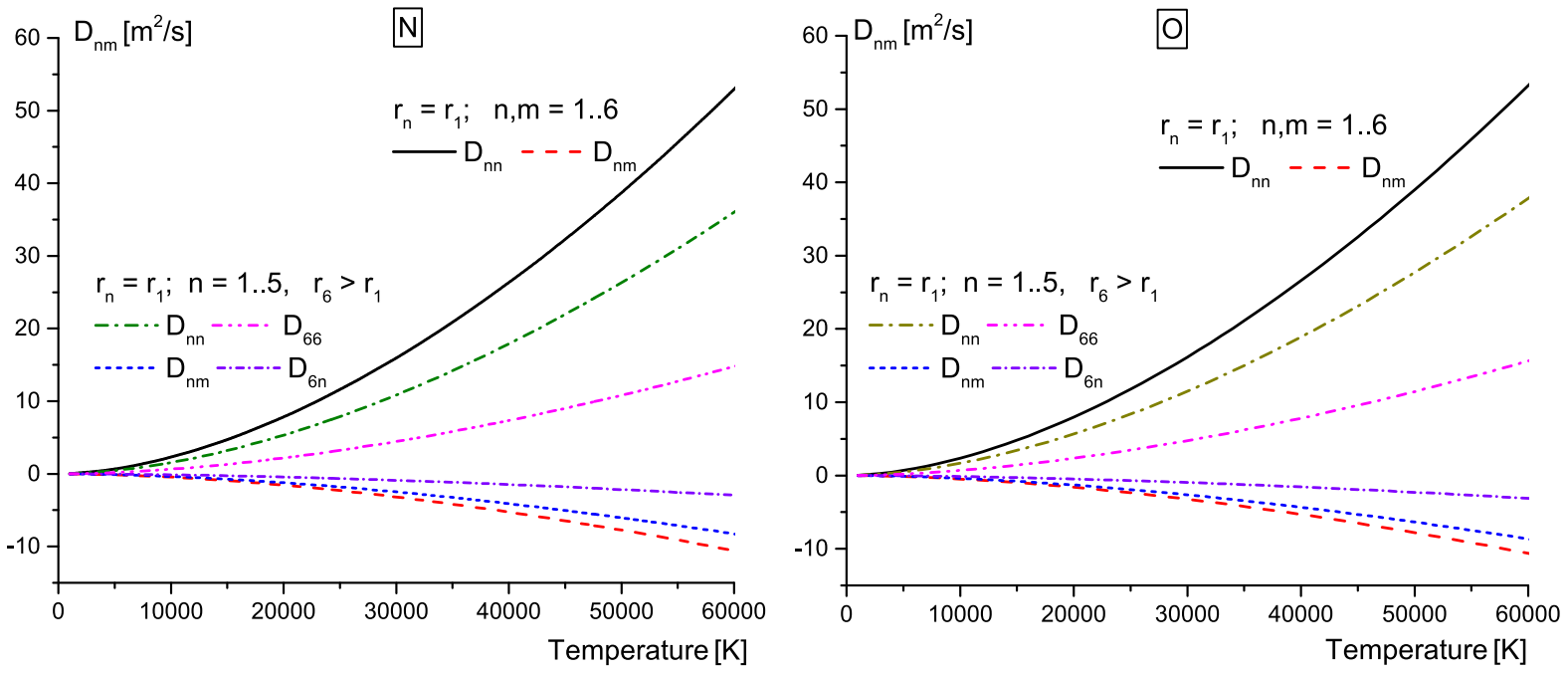

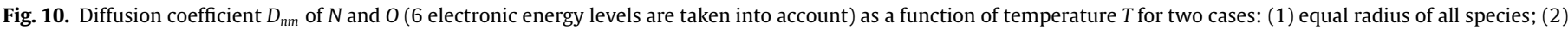
radius of species on 6th level differs. 

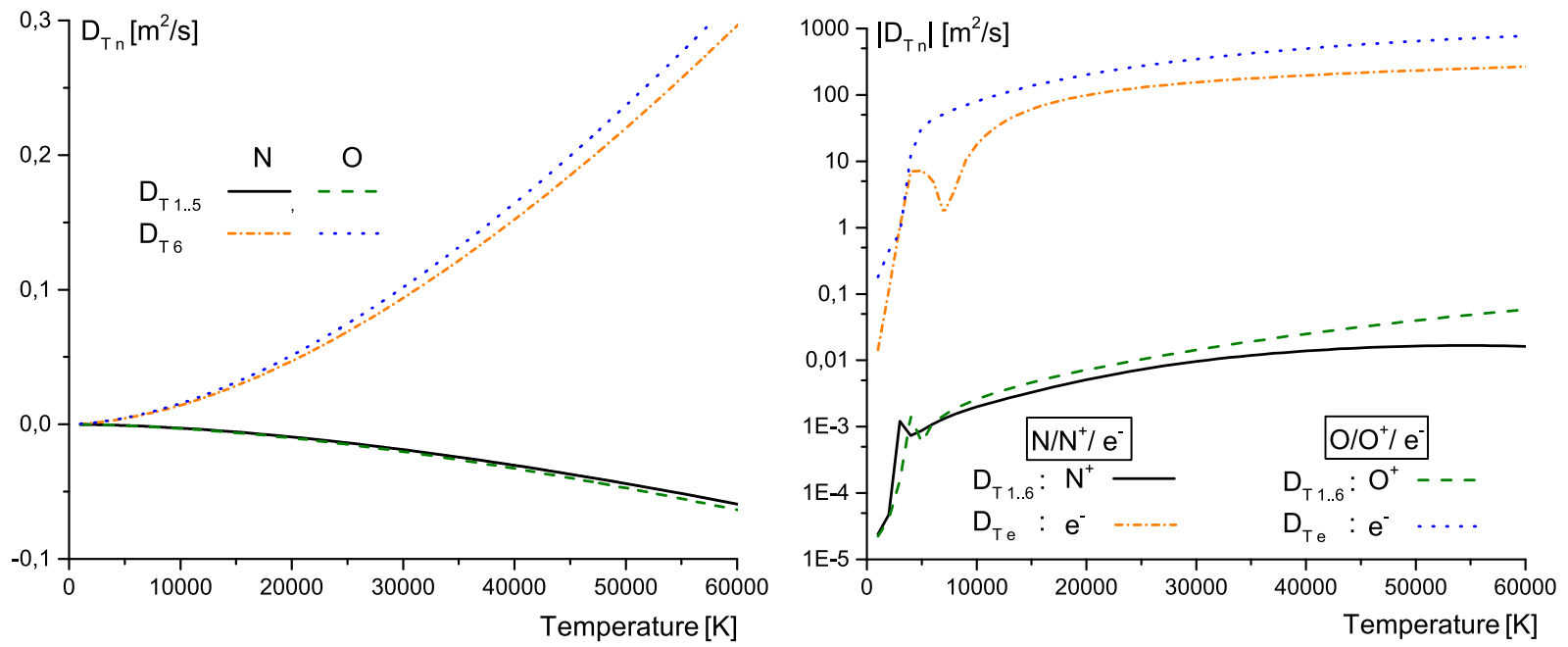

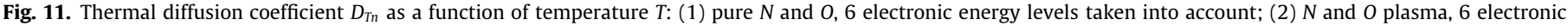
energy levels of neutral and ionized atoms are taken into account.
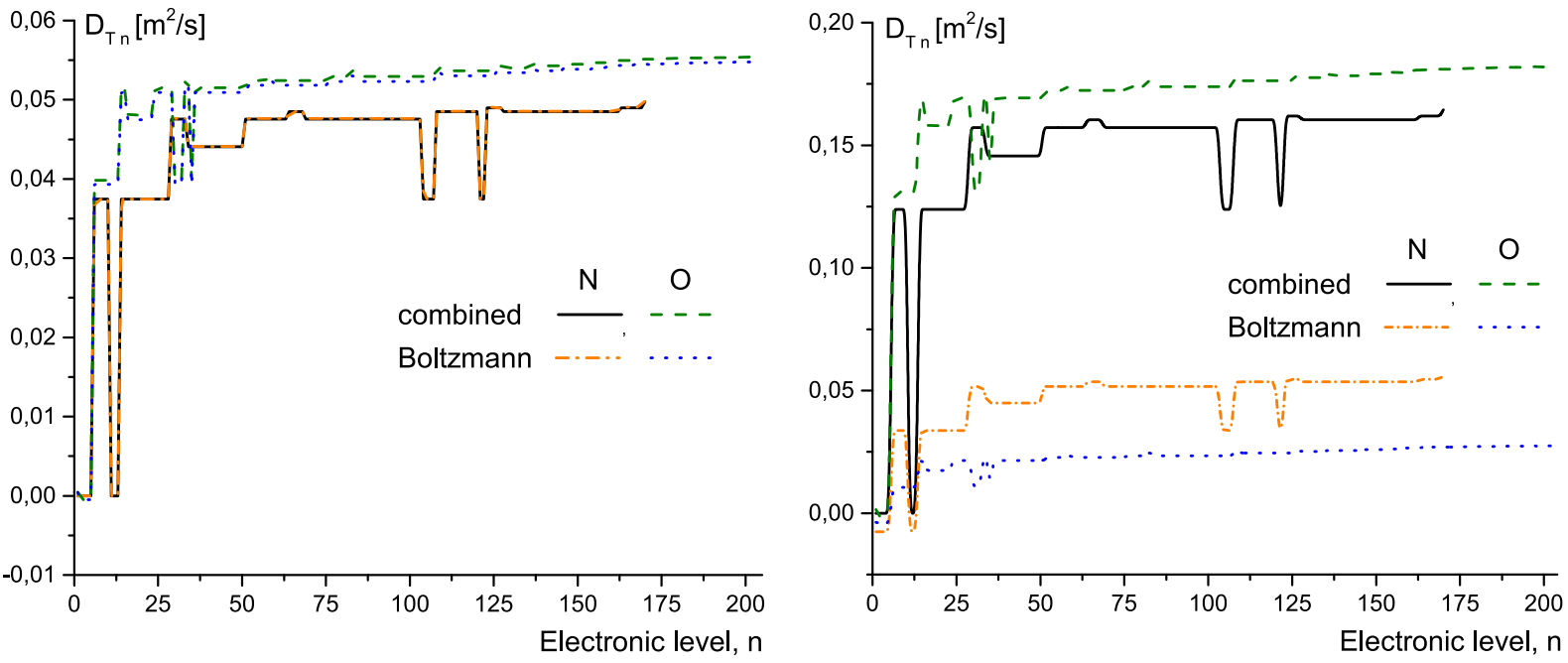

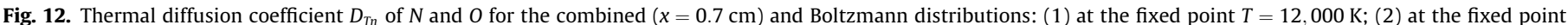
$T=24,000 \mathrm{~K}$.

over increasing atomic radius enhances this effect. However, thermal diffusion coefficients of ions are still much lower than $D_{T e}$ of electrons.

In Fig. 12 the dependence of thermal diffusion coefficient $D_{T n}$ (as a function of electronic energy level) on different distributions over electronic energy states is represented. Boltzmann and combined distributions are used for the calculation for two fixed points $T=12,000 \mathrm{~K}$ and $T=24,000 \mathrm{~K}$ behind the shock wave. For $T=12,000 \mathrm{~K}$, the results of $D_{T n}$ for two distributions are nearly coincide, while at $T=24,000 \mathrm{~K} D_{T n}$ for levels higher than 10 are 3 times higher for $N$ and 5 times higher for $O$ correspondingly. Thus the distribution over electronic energy levels behind the shock front may play an important role in the case of increasing atomic radius not through the thermal conductivity, which nearly coincides with that calculated for the ground state for combined distribution, but via the channel of thermal diffusion, which is undetermined for pure atomic gas in the one-temperature approach (i.e. for the ground-state model).

\section{Conclusions}

A theoretical model for the state-specific transport coefficients in neutral and ionized atomic gas mixture flows with electronically excited states is developed. Different cutoff criteria for electronically excited states are considered, and the maximum allowed atomic radius are calculated under various conditions. The influence of increasing atomic size on the transport properties of high-temperature flows is evaluated. It is shown that the Slater's approach can be used for preliminary estimations of the stateresolved transport coefficients in pure atomic gases. It is found that for atomic oxygen $O$, the diameter of an excited atom increases faster than for $N$, which, in turn, affects the transport coefficients of $O$. Effective collision cross-section are calculated for atoms with varying size. For ionized mixtures resonant charge-transfer process is taken into account while computing cross-sections.

Different distributions on electronic energy levels, such as the Boltzmann distribution, modified for electronic excitation Saha 
distribution, and combination of these two distributions are implemented for the calculation of transport properties.

State-dependent diffusion coefficients of atomic gases with electronic degrees of freedom are investigated. The influence of atomic size on diffusion coefficients is pointed out. It is shown that similarly to molecular vibrationally excited gases in state-to-state approach, for electronically excited atomic gas cross diffusion coefficients satisfy the same rule: $D_{n m}=D_{m n}(n \neq m)$. Thermal diffusion coefficients of neutral and ionized gases are calculated. While the weak influence of increasing radius is found for the case of six low-lying electronic levels, the role of distribution occurs more important. Thus thermal diffusion coefficients of each electronic level strongly depend on the distribution over electronic energy levels and therefore can modify the heat flux and diffusion velocity.

Using of the state-to-state and one-temperature (ground-state) models and different number of excited levels yields practically the same partial thermal conductivity and shear viscosity coefficients in the temperature range $1000-14,000 \mathrm{~K}$. Beyond $14,000 \mathrm{~K}$ the increasing radius of excited atoms causes a strong decrease in the transport coefficients calculated on the basis of the Boltzmann distributions, especially for the case when all electronic states are considered. The charge-transfer process influences mostly the shear viscosity coefficient and weakly affects the thermal conductivity coefficient. It is worth mentioning that in the high-temperature region just behind the shock front, the populations of highlying levels do not follow the Boltzmann distribution, molar fractions of high-lying states are quite low, and, consequently, the increased atomic size does not affect the flow around reentering vehicles. Therefore it justifies implementation of simplified transport models for hypersonic flow simulations. However in some plasma applications with different distributions over electronically excited states the state-specific transport coefficients may considerably modify the predicted heat and mass transfer.

\section{Acknowledgement}

This study is supported by the Russian Foundation for Basic Research, project 16-38-60009, and Saint Petersburg State University, project 6.37.206.2016.

\section{References}

[1] J. Hirschfelder, Heat conductivity in polyatomic or electrically excited gases, J. Chem. Phys. 26 (1957) 282-285.

[2] M. Capitelli, Transport properties of partially ionized gases, Journal de Physique, Colloque 38 (C3) (1977), C3-227-C3-237 (supplement au num. 8).

[3] I. Sokolova, Math. Mod. 1 (3) (1989) 84-99.

[4] D. Bruno, A. Laricchiuta, M. Capitelli, C. Catalfamo, Effect of electronic excited states on transport in magnetized hydrogen plasma, Phys. Plasmas 14 (2007) 022303.

[5] D. Bruno, M. Capitelli, C. Catalfamo, A. Laricchiuta, Transport of internal electronic energy in atomic hydrogen thermal plasmas, Phys. Plasmas 14 (2007) 072308

[6] D. Bruno, M. Capitelli, C. Catalfamo, A. Laricchiuta, Cutoff criteria of electronic partition functions and transport properties of atomic hydrogen thermal plasmas, Phys. Plasmas 15 (2008) 112306.

[7] E. Kustova, L. Puzyreva, Transport coefficients in nonequilibrium gas-mixture flows with electronic excitation, Phys. Rev. E 80 (4) (2009) 046407.

[8] K. Singh, G. Singh, R. Sharma, Role of electronic excitation on thermodynamic and transport properties of argon and argon-hydrogen plasmas, Phys. Plasmas 17 (7) (2010) 072309.

[9] V. Istomin, E. Kustova, L. Puzyreva, Transport properties of electronically excited $\mathrm{N}_{2} / \mathrm{N}$ and $\mathrm{O}_{2} / \mathrm{O}$ mixtures, in: D. Levin et al. (Eds.), Rarefied Gas Dynamics, Aip Conference Proceedings, vol. 1333, American Institute of Physics, Melville, Ny, 2011, pp. 667-672.

[10] V. Istomin, F Kustova, Transport properties of five-component nitrogen and oxygen ionized mixtures with electronic excitation, in: M. Mareschal, A Santos (Eds.), Rarefied Gas Dynamics, Aip Conference Proceedings, vol. 1501, American Institute of Physics, Melville, Ny, 2012, pp. 168-174.

[11] M. Capitelli, D. Bruno, A. Laricchiuta, Fundamental aspects of plasma chemical physics: transport, Springer Series on Atomic, Optical, and Plasma Physics, vol. 74, Springer Verlag, Berlin, 2013.
[12] V. Istomin, E. Kustova, Effect of electronic excitation on high-temperature flows behind strong shock waves, in: J. Fan (Ed.), Rarefied Gas Dynamics, Aip Conference Proceedings, vol. 1628, American Institute of Physics, Melville, Ny, 2014, pp. 1221-1228.

13] V.A. Istomin, E.V. Kustova, M.A. Mekhonoshina, Eucken correction in high temperature gases with electronic excitation, J. Chem. Phys. 140 (2014) 184311

[14] V.A. Istomin, E.V. Kustova, Effect of electronic excitation on high-temperature flows of ionized nitrogen and oxygen mixtures behind strong shock waves, in: Rarefied Gas Dynamics, Aip Conference Proceedings, vol. 1786, 2016, p. 150003.

[15] V.A. Istomin, E.V. Kustova, Transport coefficients and heat fluxes in nonequilibrium high-temperature flows with electronic excitation, Phys. Plasmas 24 (2) (2017), http://dx.doi.org/10.1063/1.4975315, accepted for publication,

[16] E. Nagnibeda, E. Kustova, Nonequilibrium Reacting Gas Flows. Kinetic Theory of Transport and Relaxation Processes, Springer-Verlag, Berlin, Heidelberg. 2009.

[17] A. Aliat, A. Chikhaoui, E. Kustova, Non-equilibrium kinetics of a radiative Co flow behind a shock wave, Phys. Rev. E 68 (2003) 056306.

18] A. Aliat, E. Kustova, A Chikhaoui, State-to-state reaction rates in gases with vibration-electronic-dissociation coupling: the influence on a radiative shock heated co flow, Chem. Phys. 314 (2005) 37-47.

[19] A. Guy, A. Bourdon, M.-Y. Perrin, Consistent multi-internal-temperature models for vibrational and electronic nonequilibrium in hypersonic nitrogen plasma flows, Phys. Plasmas 22 (9) (2015) 043507.

[20] V.A. Istomin, E.V. Kustova, State-to-state kinetics and transport properties of electronically excited $\mathrm{N}$ and $\mathrm{O}$ atoms, in: Rarefied Gas Dynamics, Aip Conference Proceedings, vol. 1786, 2016, p. 150002.

[21] C.O. Johnston, Nonequilibrium shock-layer radiative heating for earth and titan entry, Virginia Polytechnic Institute and State University, Virginia, USA 2006 (Ph.D. thesis).

[22] T.E. Magin, M. Panesi, A. Bourdon, A. Bultel, Analysis of the Fire Ii flight experiment by means of a collisional radiative model, Center for Turbulence Research Annual Research Briefs, Aeronautics and Aerospace Department, von Karman Institute for Fluid Dynamics, Belgium, 2008, 159-171.

[23] D.L. Cauchon, Radiative heating results from Fire Ii flight experiment at a reentry velocity of $11.4 \mathrm{~km} / \mathrm{s}$, Tech. Rep. X-1402, Nasa (Nasa Technical Memorandum).

[24] D. Bruno, M. Capitelli, C. Catalfamo, R. Celiberto, G. Colonna, P. Diomede, D Giordano, C. Gorse, A. Laricchiuta, S. Longo, D. Pagano, F. Pirani, Transport properties of high-temperature mars-atmosphere components, Esa Str 256 Esa, Esa Publications Division, Noordwijk, 2008.

[25] P. Atkins, R. Friedman (Eds.), Molecular Quantun Mechanics, 4th ed., Oxford University Press, 2005.

[26] C. Zener, Analytic atomic wave functions, Phys. Rev. 36 (1) (1930) 51-56.

[27] J.C. Slater, Atomic shielding constants, Phys. Rev. 36 (1) (1930) 57-64.

[28] V. Istomin, E. Kustova, M. Mekhonoshina, Validity of eucken formula and stokes viscosity relation in high-temperature electronically excited gases, in: J. Fan (Ed.), Rarefied gas dynamics, Aip Conference Proceedings, vol. 1628, American Institute of Physics, Melville, Ny, 2014, pp. 1229-1236.

29] F. Pirani, G.S. Maciel, D. Cappelletti, V. Aquilanti, Experimental benchmarks and phenomenology of interatomic forces: open-shell and electronic anisotropy effects, Int. Rev. Phys. Chem. 25 (1) (2006) 165-199.

[30] A. Molisch, B. Oehry (Eds.), Radiation Trapping in Atomic Vapours, Oxford University Press, 1998

[31] J. Neukamner, H. Rinenberg, K. Vietzke, Spectroscopy of rydberg atoms at $\mathrm{n}=500$, Phys. Rev. Lett. 59 (1987) 26.

[32] M. Capitelli, G. Colonna, A. D’Angola, Fundamental aspects of plasma chemical physics: thermodynamics, Springer Series on Atomic, Optical, and Plasma Physics, vol. 66, Springer Verlag, Berlin, 2012.

[33] M. Panesi, Physical Models for Nonequilibrium Plasma Flow Simulations at High Speed Re-entry Conditions, von Karman Institute, Rhode-Saint-Genese, Belgium, 2009 (Ph.D. thesis).

[34] A. Guy, Collisional-Radiative and Macroscopic Models for the Thermochemica Relaxation of Non-equilibrium Hypersonic Lows, Ecole Centrale Paris, Paris, France, 2013 (Ph.D. thesis).

[35] J. Ferziger, H. Kaper, Mathematical Theory of Transport Processes in Gases, North-Holland, Amsterdam, London, 1972.

[36] E. Clementi, D.L. Raimondi, Atomic screening constants from scf functions, J. Chem. Phys. 38 (11) (1963) 2686-2689.

[37] J. Stallcop, H. Partridge, A. Pradhan, E. Levin, Potential energies and collision integrals for interactions of carbon and nitrogen atoms, J. Thermophys. Heat Transfer 14 (4) (2000) 480-488.

[38] M.J. Wright, H.H. Hwang, D.W. Schwenke, Recommended collision integrals for transport property computations. Part 2: Mars and Venus entries, Aiaa J. 45 (1) (2007) 281-288.

[39] J.R.A. Kramida, Yu. Ralchenko, N.A. Team, Nist Atomic Spectra Database (ver. 5.3), [Online], National Institute of Standards and Technology, Gaithersburg, Md, 2015.

[40] C.E. Moore, Tables of Spectra of Hydrogen, Carbon, Nitrogen, and Oxygen Atoms and Ions, in Crc Series in Evaluated Data in Atomic Physics, Crc Press, Boca Raton, Fl, 1993.

[41] M. Capitelli, G. Colonna, D. Giordano, L. Marraffa, A. Casavola, P. Minelli, D. Pagano, L. Pietanza, F. Taccogna, Tables of Internal Partition Functions and Thermodynamic Properties of High-Temperature Mars-Atmosphere Species from 50 K to 50,000 K, Esa str-246, Esa Publications Division, Estec, Noordwijk, The Netherlands, 2005. 
[42] M. Panesi, T.E. Magin, A. Bourdon, A. Bultel, O. Chazot, Fire Ii flight experiment analysis by means of a collisional-radiative model, J. Thermophys. Heat Transfer 23 (2) (2009) 236-248.

[43] J.-P. Sarrette, A.-M. Gomes, J. Bacri, C. Laux, C. Kruger, Collisional-radiative modeling of quasi-thermal air plasmas with electronic temperatures between 2000 and 13,000 K, J. Quant. Spectrosc. Radiat. Transfer 1 (2) (2001) 53-125.

[44] P. Teulet, J.-P. Sarrette, A.-M. Gomes, Collision-radiative modeling of one- and two-temperature air and air-sodium plasmas at atmospheric pressure with temperatures of 2000-12,000 K, J. Quant. Spectrosc. Radiat. Transfer 1 (2001) 70-159.

[45] A. Bultel, B. Cheron, A. Bourdon, O. Motapon, I. Schneider, Collisional-radiative model in air for earth re-entry problems, Phys, Plasmas 13 (4) (2006) 11.

[46] O. Kunova, E. Kustova, M. Mekhonoshina, E. Nagnibeda, Non-equilibrium kinetics, diffusion and heat transfer in shock heated flows of $\mathrm{N}_{2} / \mathrm{N}$ and $\mathrm{O}_{2} / \mathrm{O}$ mixtures, Chem. Phys. 463 (2015) 70-81.

[47] S. Auerbach, R. Cohen, J. Gilmer, A. Mirin, M. Rensink, Plasma transport caused by ion/ neutral atom collisions, Nucl. Fusion 10 (1984) 1251-1257.

[48] E. Kustova, E. Nagnibeda, T. Alexandrova, A. Chikhaoui, On the nonequilibrium kinetics and heat transfer in nozzle flows, Chem. Phys. 276 (2) (2002) 139-154.
[49] I. Armenise, E. Kustova, On different contributions to the heat flux and diffusion in non-equilibrium flows, Chem. Phys. 428 (2014) 90-104.

[50] E. Josyula, J. Burt, E. Kustova, P. Vedula, Influence of state-to-state transport coefficients on surface heat transfer in hypersonic flows, Aiaa Paper 20140864, doi:10.2514/6.2014-0864.

[51] E. Josyula, J. Burt, E. Kustova, P. Vedula, M. Mekhonoshina, State-to-state kinetic modeling of dissociating and radiating hypersonic flows, Aiaa Paper 2015-0475, doi:10.2514/6.2015-0475.

[52] E. Kustova, On the simplified state-to-state transport coefficients, Chem. Phys. 270 (1) (2001) 177-195.

[53] E.V. Kustova, M.A. Mekhonoshina, Similarity criteria in vibrationally and electronically excited gases, in: Rarefied Gas Dynamics, Aip Conference Proceedings, vol. 1786, 2016, p. 150006.

[54] V.E. Gollant, A.P. Zhilnskiy, S.A. Sakharov, Fundamentals of Plasma Physics, Atomizdat, Moscow, 1977, in Russian.

[55] J.D. Ramshaw, C.H. Chang, Ambipolar diffusion in two-temperature multicomponent plasmas, Plasma Chem. Plasma Process. 13 (3) (1993) 489498. 